\title{
El crecimiento cantonal en el Ecuador y el papel de la heterogeneidad espacial
}

\author{
Nicola Pontarollo ${ }^{1}$, Rodrigo Mendieta y Diego Ontaneda
}

\section{Resumen}

En este documento se identifican los determinantes del crecimiento del valor agregado bruto (VAB) per cápita en el Ecuador durante el período 2007-2015, utilizando una extensión espacial del modelo de Mankiw, Romer y Weil (MRW). Debido a que el Ecuador es un país caracterizado por profundos desequilibrios socioeconómicos a nivel de los territorios, las estimaciones con técnicas clásicas que miden efectos medios o "globales" no serían tan justificables y tendrían escasas implicaciones políticas. En consecuencia, en el presente artículo se utiliza la técnica de filtrado espacial, que es una evolución reciente de la regresión geográficamente ponderada (GWR), para tomar en cuenta la heterogeneidad espacial de los coeficientes de una regresión de crecimiento que considera explícitamente tanto el capital físico como humano. Los resultados muestran que los cantones ecuatorianos tienen una amplia gama de tasas de convergencia y que el efecto del capital físico y humano varía en el espacio.

\section{Palabras clave}

Crecimiento económico, análisis económico, desarrollo regional, economía regional, modelos econométricos, indicadores del desarrollo, Ecuador

\section{Clasificación JEL}

C21, O47, R11

\section{Autores}

Nicola Pontarollo es Oficial de Estadísticas de la Dirección de Competencias, Unidad de Monitoreo, Indicadores y Evaluación de Impacto del Centro Común de Investigación (JRC) de la Comisión Europea (Ispra, Italia). Correo electrónico: nicola. pontarollo@ec.europa.eu.

Rodrigo Mendieta Muñoz es Coordinador del Grupo de Investigación en Economía Regional en la Facultad de Ciencias Económicas y Administrativas de la Universidad de Cuenca (Ecuador). Correo electrónico: rodrigo.mendieta@ucuenca.edu.ec.

Diego Ontaneda Jiménez es Investigador del Grupo de Investigación en Economía Regional en la Facultad de Ciencias Económicas y Administrativas de la Universidad de Cuenca (Ecuador). Correo electrónico: diego.ontanedaj@ucuenca.edu.ec.

\footnotetext{
Ni la Comisión Europea ni ninguna persona que actúe en su nombre son responsables del uso que pudiera hacerse de la información contenida en esta publicación. Los puntos de vista expresados en este estudio son de los autores y no reflejan necesariamente los puntos de vista de la Comisión Europea.
} 


\section{Introducción}

Este trabajo tiene por objeto estimar los determinantes del crecimiento económico cantonal en el Ecuador sobre la base de un modelo de convergencia $\beta$ (Baumol, 1986; Barro y Sala-i-Martin, 1992; Mankiw, Romer y Weil, 1992) para 214 cantones durante el período 2007-2015². Para ello se utiliza una técnica espacial alternativa llamada "filtrado espacial" (Griffith, 2008), a fin de abordar tanto la dependencia espacial como la heterogeneidad en los impactos de las variables independientes.

En comparación con otras áreas del mundo, la literatura sobre crecimiento económico subnacional en América Latina es más bien escasa ${ }^{3}$. En el caso ecuatoriano, pocos son los autores que han medido la convergencia y los determinantes del crecimiento económico, dado que el análisis ha estado condicionado a la falta de disponibilidad de información económica confiable a nivel provincial y cantonal hasta 2006. En uno de los primeros estudios que se han realizado sobre el caso ecuatoriano, Mendieta Muñoz (2015) encuentra convergencia condicional entre 2007 y 2012 usando datos cantonales. En esta misma línea, Ramón-Mendieta, Ochoa-Moreno y Ochoa-Jiménez (2013), sobre la base de datos provinciales desde 1993 hasta 2011, concluyen que en el Ecuador hay convergencia regional. Sin embargo, este proceso no es suficiente para reducir las disparidades regionales. Por otra parte, usando técnicas econométricas espaciales, los resultados muestran que el proceso de convergencia está presente, pero involucra únicamente al clúster de cantones más desarrollados (Mendieta Muñoz y Pontarollo, 2016). Al igual que en los estudios previos, Szeles y Mendieta Muñoz (2016), haciendo uso de modelos paramétricos y no paramétricos, encuentran evidencia de convergencia absoluta y condicional, tanto a nivel cantonal como provincial, para el período 2007-2014.

Este estudio se distingue de los anteriores porque estima el modelo de convergencia $\beta$ usando una técnica econométrica - los filtros espaciales -, que toma en cuenta la dependencia espacial entre las economías cantonales, pero, al mismo tiempo, permite que las variables incluidas en el modelo tengan un efecto diferenciado entre cantones, debido a las diferencias en cuanto a estructuras productivas y de contexto. De esta forma, no es necesario asumir, como en algunos análisis previos, que todos los cantones respondan de la misma forma a las variables que condicionan el crecimiento, lo que es plausible en el caso de que existan diferencias estructurales entre ellos.

Con respecto a la dependencia espacial, en muchos estudios, entre ellos los de Fingleton (1999) y Le Gallo y Ertur (2003), se ha demostrado la presencia de correlación espacial en los residuos de los modelos de crecimiento estimados tradicionalmente. Esto conduce a la inferencia incorrecta en las estimaciones de parámetros significativos (modelo de error espacial) o a estimaciones de los parámetros tanto sesgadas como ineficientes (modelo de rezago espacial). La técnica de filtrado espacial nos permite solucionar el problema de los residuos espacialmente autocorrelacionados tomando en cuenta los efectos de la interacción espacial que tiene lugar entre las variables, y, a la vez, permite considerar los efectos de los desbordamientos espaciales (Griffith, 2003). Además, al ser una

\footnotetext{
2 Se excluyen del análisis los municipios de Putumayo, Shushufindi, Cuyabeno, Orellana, La Joya de los Sachas, Sevilla de Oro y Quinsaloma debido a que son valores atípicos del VAB al provenir principalmente de la extracción de minerales.

3 Entre los países donde existe mayor evidencia empírica se encuentran el Brasil, Colombia y México. Esquivel (1999) y GomezZaldívar, Ventosa-Santaulària y Wallace (2012) encuentran evidencia de convergencia entre regiones mexicanas desde 1940 hasta 1995, y desde 1940 hasta 2009, respectivamente, mientras que Rodríguez-Pose y Villarreal (2015) centran su atención en el papel de las condiciones socioeconómicas en función del crecimiento. Cárdenas y Ponton (1995), así como Gómez y Santana (2016), se enfocan en la convergencia regional colombiana y también encuentran evidencia de convergencia. Royuela y García (2015) confirman que existe convergencia en Colombia en variables sociales clave, aunque no en la clásica variable económica (PIB per cápita), y que la autocorrelación espacial refuerza los procesos de convergencia. En el caso del Brasil, Azzoni (2001) señala que ha habido convergencia regional entre 1939 y 1995, pero la desigualdad ha cambiado en el transcurso del tiempo. Por último, De Andrade Lima y Silveira Neto (2016) descubren que existe una fuerte dependencia espacial entre las microrregiones brasileñas y que las inversiones, tanto en capital físico como en capital humano, son importantes para el crecimiento de las economías regionales del Brasil. Este resultado ha sido confirmado por Resende y otros (2016).
} 
extensión de la GWR (Fotheringham, Brunsdon y Charlton, 2002) propuesta por Griffith (2008), esta técnica permite estimar diferentes coeficientes locales y no un único coeficiente para cada variable como en el caso del método de mínimos cuadrados ordinarios.

En la sección II se describe la dinámica espacial del VAB per cápita. En la sección III se señala el modelo empírico utilizado en el estudio del crecimiento y se describe el modelo espacial utilizado. En la sección IV se discuten los resultados y, por último, en la sección $V$ se presentan las conclusiones.

\section{Dinámicas espaciales del crecimiento regional en el Ecuador}

Un primer análisis de la dinámica espacial con respecto al caso del Ecuador se basa en el Índice de Moran (IM) (Moran, 1950), que es una de las medidas más utilizadas para detectar y medir la fuerza de la dependencia (autocorrelación) espacial. Esencialmente, el Índice de Moran relaciona el valor de una variable determinada con su rezago espacial, esto es, el valor que asume dicha variable en las ubicaciones vecinas. Así, el Índice de Moran puede definirse de la siguiente forma:

$$
I M=\frac{N}{\sum_{i} \sum_{j} w_{i j}} \frac{\sum_{i} \sum_{j} w_{i j}\left(x_{i}-\bar{x}\right)\left(x_{i}-\bar{x}\right)}{\sum_{i}\left(x_{i}-\bar{x}\right)}
$$

Donde $N$ es el número de unidades en el mapa (es decir, áreas o puntos); $x$ es la variable de interés; $\bar{x}$ es la media de $x$, y $w_{i j}$ es un elemento de la matriz de pesos espaciales $W_{i j}$, donde $j$ representa el conjunto de las regiones vecinas a $i$. El Índice de Moran en general oscila entre el máximo y el mínimo autovalor. En el caso de una matriz estandarizada por fila, oscila entre -1 y 1. Un coeficiente positivo indica una autocorrelación espacial positiva, es decir, en el mapa pueden distinguirse clústeres de valores similares. El caso contrario representa regímenes de asociación espacial negativa, esto es, valores disímiles se encuentran agrupados juntos en el mapa. Un valor de Índice de Moran próximo a 0 indica un patrón espacial aleatorio.

Siguiendo la primera ley de la Geografía de Tobler (1970), que señala que todos los lugares están relacionados, pero los lugares cercanos están más relacionados que los lugares lejanos, cada elemento $i$ de la matriz de pesos espaciales $W_{i j}$ se considera vecino a todos los demás cantones j, pero el peso de los vecinos es proporcional al inverso de la distancia al cuadrado entre los centroides.

Esto evita tener que seleccionar matrices de pesos espaciales ad hoc que se basan en maximizar el criterio de información de Akaike sin tener en cuenta las potenciales razones por las cuales en la práctica tiene más sentido una definición sobre otra, como hacen, por ejemplo, Arbia, Battisti y Di Vaio (2010), y Postiglione, Andreano y Benedetti (2017). Además, permite que haya una mayor interacción entre dos cantones que tienen una distancia menor entre sus centroides que entre aquellos que tienen una distancia mayor.

En el eje izquierdo del gráfico 1 se presenta la denominada "convergencia sigma", es decir, la dispersión del (In) del VAB per cápita4 ${ }^{4}$ En el eje derecho, en tanto, se muestra el Índice de Moran de

\footnotetext{
4 La convergencia tipo sigma es una medida de dispersión de la renta de los territorios y muestra cómo, a medida que transcurre el tiempo, las diferencias entre las economías tienden a disminuir. Esto implica que las disparidades entre los territorios tenderán a reducirse con el tiempo y, por lo tanto, se irán aproximando hacia un único estado estacionario. Siguiendo el enfoque propuesto por Barro y Sala-i-Martin (1992), la convergencia tipo sigma puede medirse como la desviación estándar del logaritmo de la renta per cápita.
} 
la misma variable durante el período $2007-2015^{5}$. El valor p del Índice de Moran obtenido a través de 1.000 aleatorizaciones es significativo para todos los años considerados. Se debe señalar que el Índice de Moran crece muy levemente durante el período, mientras la convergencia sigma muestra una tendencia creciente mucho más marcada en el tiempo. Estos patrones implican que la disparidad intercantonal del VAB per cápita se ha ido incrementando durante el período de análisis, mientras que la polarización se mantiene estable.

\section{Gráfico 1}

Ecuador: convergencia sigma e Índice de Moran

del log del valor agregado bruto (VAB) per cápita cantonal, 2007-2015

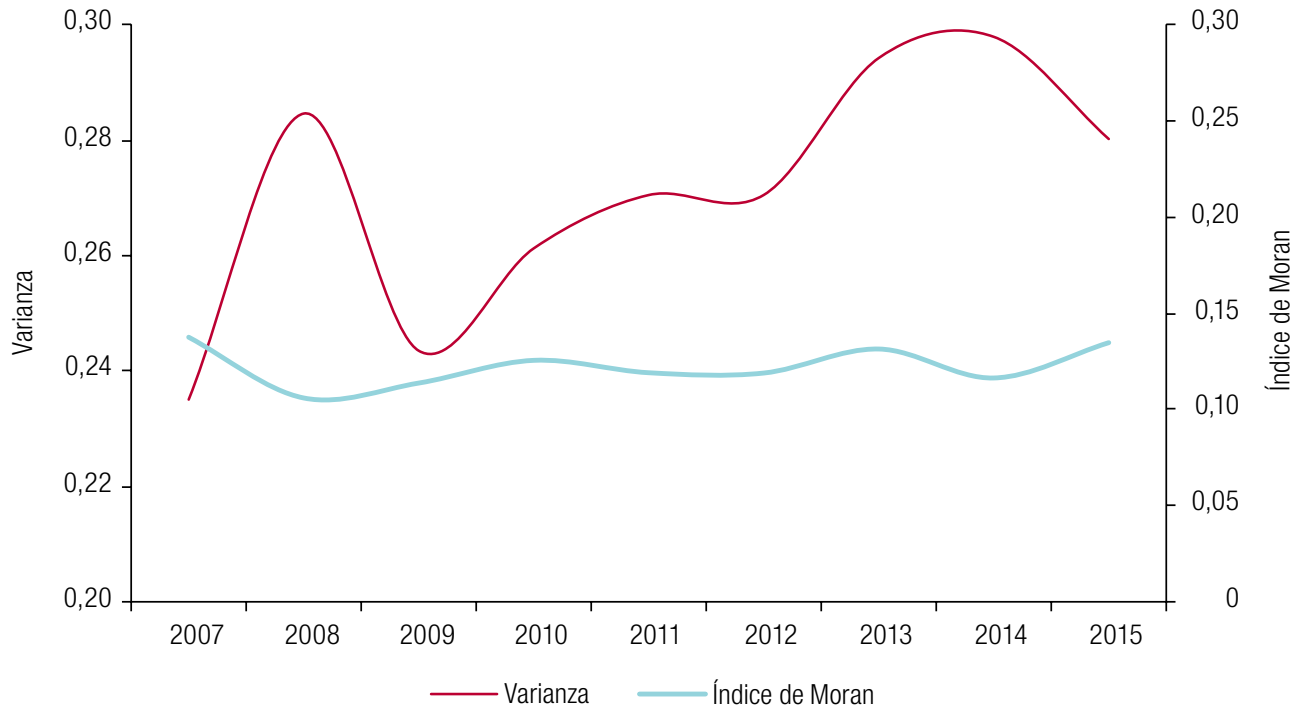

Fuente: Elaboración propia.

El análisis se complementa con los mapas de los índices de autocorrelación espacial local que tienen como finalidad la detección de aglomeraciones (clústeres), lo que no es posible lograr con las medidas de asociación espacial global. Esto implica que, aunque los contrastes globales hayan detectado un determinado esquema de autocorrelación espacial, este puede no mantenerse para toda la muestra. En tanto, el análisis local examina particularmente subregiones donde se determina si dicha área representa clústeres de valores altos (hot spot) o de valores bajos (cold spot) (Getis y Fischer, 2009; Celebioglu y Dall'erba, 2010; Cravo y Resende, 2013).

La expresión del estadístico de Moran local (Anselin, 1995) viene dada por:

$$
I_{i}=\frac{\left(x_{i}-\bar{x}\right)}{\sum_{i} \frac{\left(x_{i}-\bar{x}\right)^{2}}{N}} \sum_{j \in J_{i}} w_{i j}\left(x_{j}-\bar{x}\right)
$$

El estadístico de Moran cumple con dos requerimientos: i) cuantifica el grado de agrupamiento significativo de valores similares alrededor de una observación y ii) cumple con que la suma del indicador para todas las observaciones es proporcional al indicador global de asociación espacial. Los valores de p del estadístico de Moran local se basan en la corrección de Bonferroni.

\footnotetext{
5 EI VAB per cápita se obtuvo a partir de los repositorios de datos del Banco Central del Ecuador (BCE) y de las estadísticas poblacionales proporcionadas por el Instituto Nacional de Estadísticas y Censos (INEC).
} 
En el mapa 1 se muestra que, mientras que los cantones que forman clústeres significativos para el crecimiento son pocos, hay más cantones, y cantones más definidos, para el VAB per cápita en 2007. Los clústeres significativos alto-alto se ubican en las áreas de Quito y Guayaquil, mientras que los clústeres bajo-bajo están en el sur del país, en Cañar, y en la parte central de la zona de la costa.

\section{Mapa 1}

Ecuador: estadístico de Moran local de las variables incluidas en el modelo

A. Crecimiento del valor agregado bruto (VAB) per cápita, 2007-2015
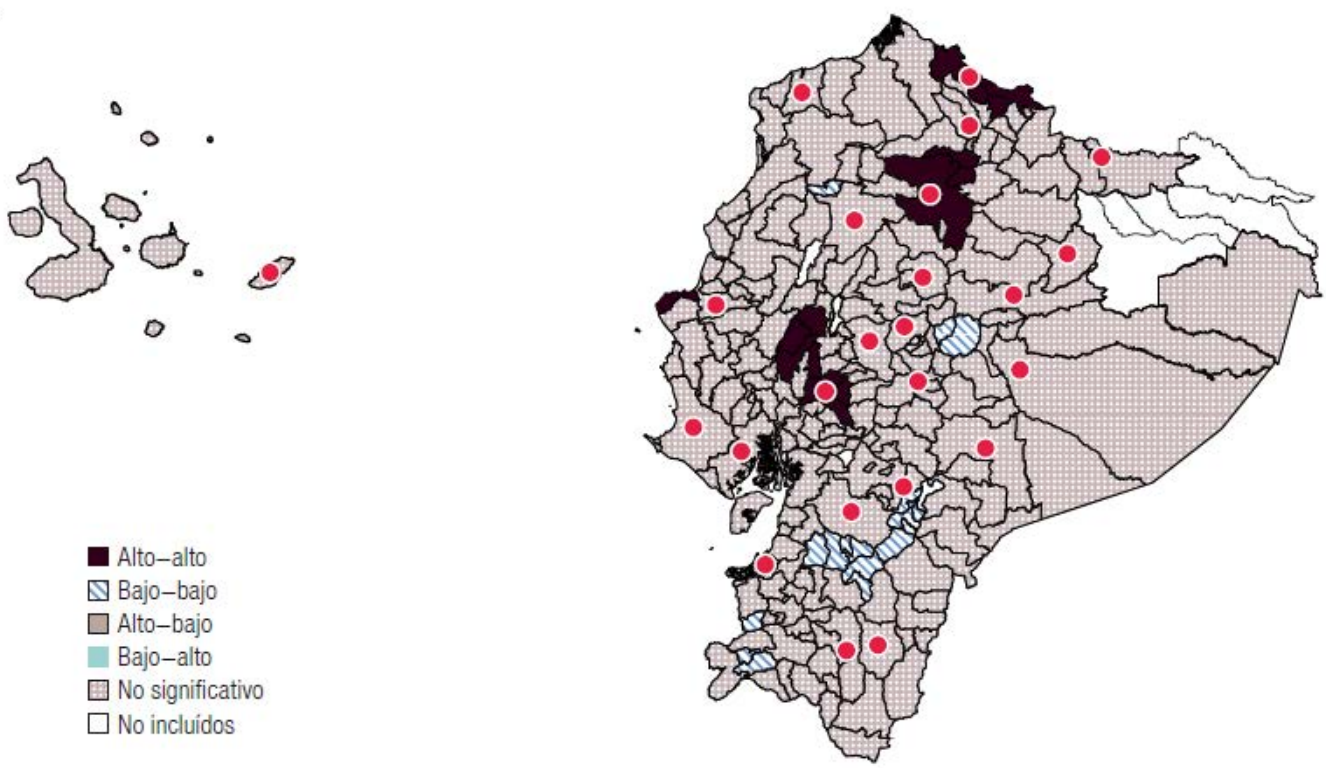

B. Valor agregado bruto (VAB) per cápita, 2007
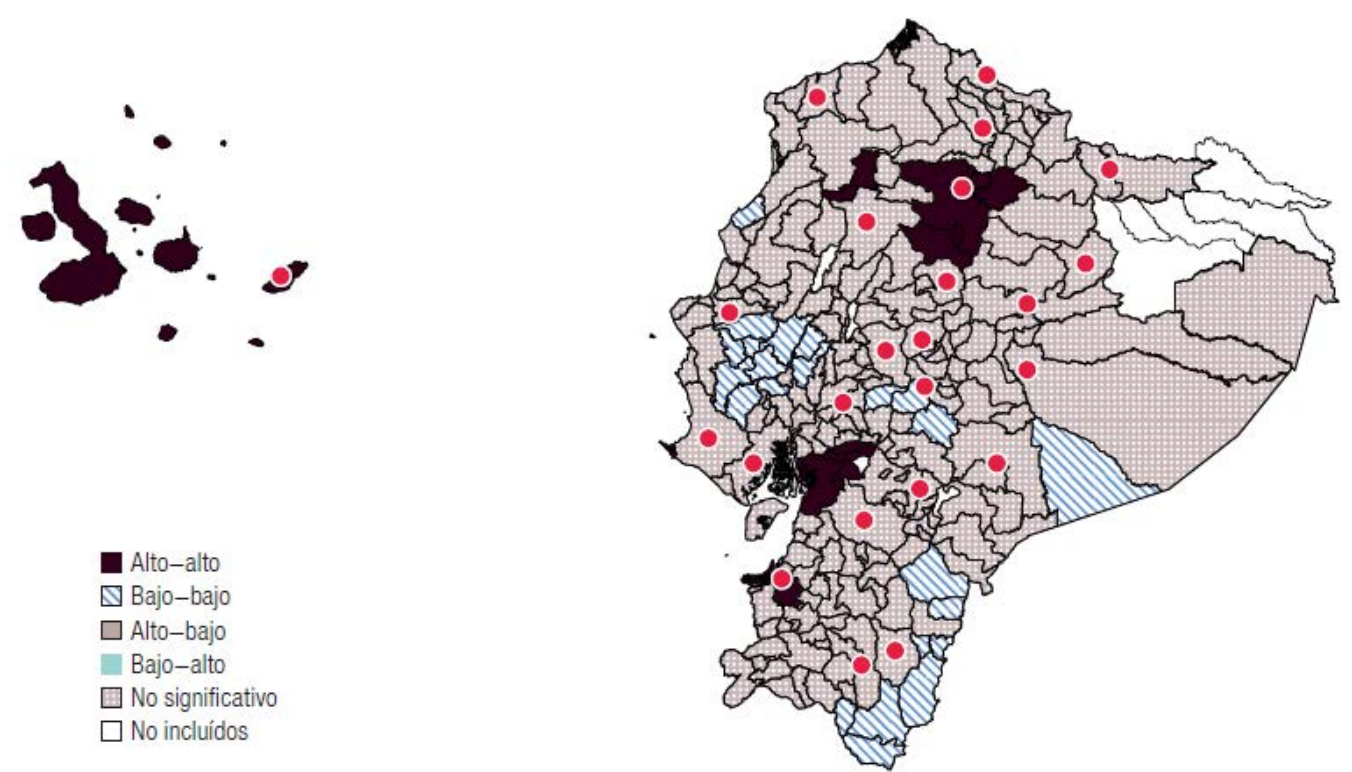

Fuente: Elaboración propia.

Nota: Los puntos representan las capitales provinciales. 


\section{El modelo teórico, datos y metodología espacial}

\section{El modelo teórico y los datos}

Nuestro modelo empírico se basa en el concepto de convergencia $\beta$ condicional, que se contrasta mediante el siguiente modelo econométrico de sección cruzada (Mankiw, Romer y Weil, 1992):

$$
\begin{gathered}
\frac{1}{T} \ln \left(\frac{y_{i, T}}{y_{i, 0}}\right)=\alpha_{i}+\lambda_{i} \ln \left(y_{i, 0}\right)+\pi_{i} \ln \left(n_{i}+g+\delta\right) \\
+\vartheta_{i} \ln (i n v)+\gamma_{i} \ln (h)+u_{i t}
\end{gathered}
$$

Donde $\lambda_{i}=-T^{-1}\left(1-e^{-\beta T}\right)$ y $\alpha_{i}=g_{i}-\lambda_{i}\left(\ln A_{i, 0}-\ln y_{i, \infty}^{E}\right)$

Que implica que la tasa de crecimiento promedio de la producción per cápita del territorio $i$ en el período de tiempo $T$, a la izquierda de la ecuación (3), se relaciona con su nivel de producción per cápita en el período inicial $\left(y_{i, 0}\right)$. En la constante $\alpha_{i}$ se encuentran los términos $g_{i}$ (que mide el progreso tecnológico), $A_{i, 0}$ (el nivel de eficiencia de cada trabajador) y $y_{i, \infty}^{E}$ (el estado estacionario). Para aproximar el capital humano $h$, se adoptó el logaritmo del promedio de años de escolaridad de las personas de 24 años y más que residían en un determinado cantón ${ }^{6}$. Los datos se obtuvieron a partir del Censo de Población y Vivienda 2010. El capital físico, inv, se construyó sobre la base de la información de los activos fijos reportada por las empresas durante el Censo Nacional Económico realizado en el Ecuador en 2010. Los activos fijos o propiedades, planta y equipo, se definen como activos tangibles que son usados por una empresa en la producción o el abastecimiento de bienes o servicios, y que se espera que se usen durante más de un período. $\delta$ es la tasa de depreciación, $n_{i}$ es la tasa de crecimiento de la población, y, como sugieren Mankiw, Romer y Weil (1992), $g+\delta$ se ha puesto igual a 0.05. En el anexo A2 se muestra un mapeo de las variables.

El coeficiente $\lambda_{i}$, negativo y estadísticamente significativo, se emplea para determinar el valor de $\beta$ que aproxima la velocidad de convergencia y $u_{i t}$ es el error de estimación. La velocidad de convergencia se calcula como $\beta_{i}=-\ln \left(1-\lambda_{i} T\right) / T$, mientras que la denominada half-life $\tau=\ln (2) / \beta_{i}{ }^{7}$. De acuerdo con Abreu, de Groot y Florax (2005), los errores estándar de $\beta_{i}$ se obtienen como $\sigma_{\beta i} \frac{\sigma_{\lambda i}}{T\left(1-\lambda_{i}\right)}$, donde $\sigma_{\lambda_{i}}$ corresponde a los errores estándar de $\lambda_{i}$ estimados de acuerdo con Dawson y Richter (2006) y $T$ es el número de años. Una vez obtenidos los errores es posible conseguir fácilmente los valores $t$.

En los primeros estudios sobre convergencia (Barro y Sala-i-Martin, 1992) generalmente se analiza este proceso $\sin$ añadir variables de control y asumiendo que $\alpha_{i}=\alpha$ y que $\lambda_{i}=\lambda$, es decir, que las economías tienen una estructura homogénea. Un valor negativo y estadísticamente significativo de $\lambda$ implica que las regiones atrasadas crecen más deprisa que las ricas, lo que denota aceptación de la hipótesis de convergencia absoluta a un mismo estado estacionario. Así, en la medida en que un territorio de un país aumenta los niveles de producción por persona, su tasa de crecimiento debería descender, lo que implica que los territorios pobres crecen más deprisa que los ricos y que, en el largo plazo, todos converjan, en términos per cápita, a una misma tasa de crecimiento de la renta y a un mismo nivel de capital (estado estacionario), con lo que tiende a desaparecer la disparidad económica inicial. De todos modos, como señala Solow (1999, pág. 640), no hay nada en la teoría del crecimiento

6 Esta es la variable utilizada como proxy del capital humano en De Andrade Lima y Silveira Neto (2016), De La Fuente (1994), Benhabib y Spiegel (1994), Barro y Lee (1993) y Kyriacou (1991).

7 Half-life se puede traducir como "vida media" y se refiere al número de años necesarios para eliminar la mitad de la desviación desde un valor inicial del VAB per cápita y el valor del estado estacionario de largo plazo. 
que requiera que la configuración de estado estacionario se dé de una vez y para siempre; el estado estacionario cambiará cada cierto tiempo, cuando haya grandes revoluciones tecnológicas, cambios demográficos o variaciones en la voluntad de ahorrar e invertir.

Entonces, si no existe un mismo estado estacionario $\ln y_{i, \infty}^{E}$ en el largo plazo, o si las variables estructurales afectan de forma significativa el crecimiento, se presenta la denominada "convergencia $\beta$ condicional" (Sala-i-Martin, 1994; Cuadrado-Roura, Mancha-Navarro y Garrido-Yserte, 2000), que admite que los territorios no converjan hacia un equilibrio económico común, sino hacia estados estacionarios particulares, determinados por tasas de ahorro y niveles de inversión y tecnología específicos, producto, a su vez, de una estructura económica particular.

Para estimar la convergencia $\beta$ condicional, como se muestra en la ecuación (3), consideramos una serie de variables adicionales que condicionan el patrón de crecimiento hacia el estado estacionario: capital físico, capital humano y crecimiento de la población. Este enfoque, que supone heterogeneidad de los estados estacionarios, todavía sigue suponiendo una homogeneidad de las tasas de convergencia.

Este último punto ha sido criticado por Temple (1998 y 2000), quien observa que en un modelo de corte trasversal para diversos países es muy frecuente la heterogeneidad de los parámetros, los valores extremos y los errores de medición. A este respecto, Ketteni, Mamuneas y Stengos (2007), y Fotopoulos (2012), por ejemplo, encuentran que no hay linealidad en los procesos de crecimiento económico. En cuanto a aspectos específicos regionales, en cambio, se deben considerar los problemas relacionados con la dependencia espacial (véanse, entre otros, Anselin, 1988; Rey y Montouri, 1999; Arbia, 2006) y la heterogeneidad espacial (Ertur, Le Gallo y LeSage, 2007; Pede, Florax y Lambert, 2014).

En este artículo, mediante el uso de un modelo que explora la técnica de filtración espacial explicada en la sección III.2, se considera la eventual presencia de heterogeneidad tanto de la tasa de convergencia, es decir $\lambda_{i}=\lambda$, como en las variables que condicionan el crecimiento (Durlauf, Johnson y Temple, 2005). En comparación con el artículo de Cravo y Resende (2013), donde se utiliza la estadística de Getis (1995) para remover el componente espacial desde cada variable, aquí se admite la posibilidad de que haya no solo autocorrelación, sino también no estacionariedad, como se explica más adelante.

\section{El modelo espacial}

Los fenómenos espaciales tienen una gran importancia, sobre todo cuando consideramos tanto los factores socioeconómicos (Bockstael, 1996; Weinhold, 2002), como las implicaciones para los encargados de formular políticas (Lacombe, 2004). La presencia de patrones espaciales que afectan positiva o negativamente las variables económicas requiere una evaluación rigurosa y sistemática de su impacto y de su forma. Los filtros espaciales constituyen una perspectiva nueva e interesante de investigación del análisis econométrico espacial. Se trata de una herramienta que permite dividir las variables georreferenciadas en dos componentes - espacial y no espacial-, destacando el componente de autocorrelación espacial.

La técnica de filtración propuesta por Griffith (2003) se sirve de la técnica de descomposición de la matriz en sus vectores y valores propios, y permite la extracción de la matriz $n \times n$ de componentes ortogonales numéricos incorrelacionados (Tiefelsdorf y Boots, 1995).

Este enfoque no paramétrico tiene por objeto controlar la presencia de autocorrelación espacial mediante la introducción de variables - los vectores propios-, que no se pueden usar como predictores en lugar de las variables que no se consideran explícitamente en el modelo (Fischer y Griffith, 2008). Comparado con la técnica de Getis (1995), que filtra por separado cada variable 
dividiendo el componente espacial del no espacial, existe la ventaja de que se pueden usar variables no negativas (Griffith, 2010), lo que permite incluir, por ejemplo, tasas de crecimiento en el análisis.

El modelo se deriva de la forma matricial del Índice de Moran definida en la ecuación (1):

$$
M I=\frac{n}{1^{t} W 1} \frac{Y^{t} M W M Y}{Y^{t} M Y}
$$

donde $W$ es la matriz de los pesos espaciales, $n$ es el número de cantones, $Y$ es el vector de los valores y $M=\left(I-\frac{11^{t}}{n}\right)$ es la matriz en la cual $I$ es la matriz identidad de tamaño $n \times n, 1$ es el vector de unos de dimensión $n \times 1$ y el exponente $t$ es la matriz transpuesta. La peculiaridad de la matriz $M$ es que se centra en el vector $Y$.

Tiefelsdorf y Boots (1995) demuestran que cada uno de los valores $n$ propios de la expresión MWM (4) es un valor $I M$, una vez que se multiplica por el término de la izquierda de la expresión (4), es decir, $\frac{n}{1^{\prime} W 1}$.

La función propia vinculada a la matriz de contigüidad geográfica $W$ puede interpretarse como la asociación espacial latente de una variable georreferenciada (Getis y Griffith, 2002). $E_{1}$ (primer vector propio) es el conjunto de valores numéricos que tienen el mayor valor de $I M$ para la matriz de contigüidad geográfica dada, en tanto que $E_{2}$ (segundo vector propio) es el grupo de valores numéricos que tienen el mayor valor de $I M$ para cada grupo de valores numéricos no correlacionados con $E_{1}$. Esta construcción secuencial de vectores propios continúa hasta $E_{n}$, un conjunto de valores numéricos que tienen el mayor valor de $I M$ alcanzable por cualquier conjunto de valores numéricos que no están correlacionados con los vectores $n-1$ propios previos. Estos vectores $n$ propios describen la gama completa de posibles patrones espaciales ortogonales e incorrelacionados, y pueden interpretarse como un mapa resumido de las variables que describen la naturaleza (positiva o negativa) y el nivel (bajo, moderado o alto) de autocorrelación espacial.

El modelo espacial utilizado en el presente trabajo se refiere a un estudio reciente realizado por Griffith (2008), donde se propone un nuevo enfoque del modelo GWR (Fotheringham, Brundson y Charlton, 2002), basado en filtros espaciales a través de la construcción de nuevas variables creadas a partir del producto entre el filtro espacial y las variables espaciales. La técnica de filtración espacial resuelve los problemas de multicolinealidad y de falta de grados de libertad típicos de las estimaciones obtenidas por la GWR (Wheeler y Tiefelsdorf, 2005).

El modelo GWR estima una regresión "local” para cada localización en el espacio, ponderando las observaciones en función de la distancia desde la región objeto de estudio, sobre la base de la siguiente expresión:

$$
Y=\beta_{0,(u, v)} 1+\sum_{p=1}^{P} X_{p} \beta_{p,(u, v)}+\varepsilon
$$

Donde $Y$ es un vector $n \times 1$, y representa la variable dependiente, $\beta$ es el coeficiente de regresión, $X_{p}$ es un vector $n \times 1$ de valores de la variable $p, \varepsilon$ es un vector $n \times 1$ que contiene los términos de error aleatorio, y $(u, v)$ indica que los parámetros deben estimarse para cada locación cuyas coordenadas espaciales están dadas por el par de vectores $(u, v)$, asumiendo implícitamente que $Y, X$ y $\varepsilon$ están georreferenciados.

En su modelo, aquí denominado "filtración espacial-GWR" (FS-GWR), Griffith (2008) propone la inclusión de un término de interacción entre cada variable de atributo y cada vector propio candidato. Un modelo lineal normal con filtro espacial incorpora un grupo de $P$ regresores, $X_{p}=(k=1,2, \ldots, P)$, con un grupo de $k$ vectores propios seleccionados, $E_{k}=(k=1,2, \ldots, K)$, que representan diferentes modelos espaciales y tiene la siguiente forma: 


$$
\begin{gathered}
Y=\beta_{0, G W R}+\sum_{p=1}^{P} X_{p} \beta_{p, G W R} \approx \\
\approx\left(\beta_{0} 1+\sum_{k_{0}=1}^{K_{0}} E_{k_{0}} \beta_{k_{0}}\right)+\sum_{p=1}^{P}\left(\beta_{p} 1+\sum_{k_{p}=1}^{K p} E_{k_{p}} \beta_{k_{p}}\right) \cdot X_{p}
\end{gathered}
$$

Donde • denota el elemento de la multiplicación de matrices (es decir, producto Hadamard de matrices) y $k_{p}$ identifica los números de vector propio que describe la variable de atributo $p\left(k_{p}\right.$ es el número total de estos vectores). En la ecuación (6) se revela la presencia de los términos de interacción en cuestión, denominados $E_{k_{p}} \bullet X_{p}$. La suma del primer y el segundo término de la ecuación (6) devuelve la expresión del coeficiente de intercepto, mientras que la suma del tercer y el cuarto término multiplicado por $X_{p}$, da como resultado los coeficientes locales de las covariables. Entonces, reordenando los resultados obtenidos, cuando se consideran todos los $K$ vectores propios candidatos, se obtiene:

$$
Y=\beta_{0} 1+\sum_{p=1}^{P} X_{p} \cdot 1 \beta_{p}+\sum_{k=1}^{K} E_{k} \beta_{E_{k}}+\sum_{p=1}^{P} \sum_{k=1}^{K} X_{p} \cdot E_{k} \beta_{p E_{k}}+\varepsilon
$$

Donde los coeficientes de regresión representan valores globales y los vectores propios representan modificaciones locales de estos valores globales; los primeros dos términos (es decir, los coeficientes del intercepto y de las variables de atributos globales) son multiplicados por el vector 1 , que también es un vector propio de filtro espacial. Los últimos dos términos son las variaciones locales del intercepto y de las mismas variables. Más precisamente, los valores globales son los coeficientes necesarios para construir combinaciones lineales de los vectores propios con el fin de obtener los coeficientes de tipo GWR. La estimación de la ecuación (7) debe proseguirse mediante la recolección de todos los términos que contienen una variable de atributo común y después factorizar con el fin de determinar su coeficiente GWR, que será la suma correspondiente que aparece en la ecuación (6). Los coeficientes del GWR son vectores $n \times 1$. Operativamente, se procede de la siguiente forma:

1. Se calculan los vectores propios extraídos de la matriz de contigüidad $W$.

2. Se calculan todos los términos de interacción $X_{p} \bullet E_{k}$ para las $P$ covariables para los $K$ vectores propios candidatos (con $I M>0.25$ ).

3. Se seleccionan, con una regresión por etapas (stepwise regression), los términos de interacción, incluidos los vectores propios, que maximizan el valor del criterio de información de Akaike.

4. Se calcula el término de intercepto variable geográficamente determinado por los primeros dos términos de la ecuación (6).

5. Se calcula el coeficiente de covarianza variable geográficamente determinado por los últimos dos términos de la ecuación (6).

\section{Resultados empíricos}

En esta sección se exponen los resultados del modelo especificado en la sección III.

En el cuadro 1 se muestran los resultados de las estimaciones con mínimos cuadrados ordinarios y con filtración espacial de los coeficientes globales (o promedios). A fin de comparar los resultados de diferentes modelos, se usan dos tipos de filtración espacial: un primer tipo, denominado FS, donde 
se consideran interceptos individuales, como sugieren Getis y Griffith (2002), seleccionando los vectores propios con una regresión por etapas, para filtrar la autocorrelación espacial en los residuos; el segundo tipo es la técnica de filtración espacial-GWR, que añade a los interceptos individuales los vectores propios asociados a cada variable independiente.

Cuadro 1

Resultados empíricos

\begin{tabular}{|c|c|c|c|}
\hline & $\begin{array}{l}\text { Mínimos cuadrados } \\
\text { ordinarios }\end{array}$ & Filtrado espacial & Filtrado espacial GWR \\
\hline Intercepto & $\begin{array}{l}0,16838^{\star \star \star} \\
(0,06307)\end{array}$ & $\begin{array}{l}0,08086^{\star \star \star} \\
(0,06418)\end{array}$ & $\begin{array}{l}-0,04610^{\star \star \star} \\
(0,05700)\end{array}$ \\
\hline Log (VAB per cápita 2007) & $\begin{array}{l}-0,05421^{\star \star \star} \\
(0,00616)\end{array}$ & $\begin{array}{l}-0,05696^{\star \star \star} \\
(0,00555)\end{array}$ & $\begin{array}{l}-0,04803^{\star \star \star} \\
(0,00525)\end{array}$ \\
\hline Crecimiento pobl. & $\begin{array}{l}0,006587 \\
(0,01050)\end{array}$ & $\begin{array}{l}-0,01905^{\star \star \star} \\
(0,01425)\end{array}$ & $\begin{array}{l}-0,03011^{* * *} \\
(0,01431)\end{array}$ \\
\hline Educación & $\begin{array}{l}0,11562^{\star \star *} \\
(0,01713)\end{array}$ & $\begin{array}{l}0,13553^{\star * *} \\
(0,01606)\end{array}$ & $\begin{array}{l}0,15128^{\star \star \star} \\
(0,01455)\end{array}$ \\
\hline Capital & $\begin{array}{l}0,00230^{\star \star *} \\
(0,00047)\end{array}$ & $\begin{array}{l}0,00199^{\star \star *} \\
(0,00042)\end{array}$ & $\begin{array}{l}0,00159^{\star * \star} \\
(0,00040)\end{array}$ \\
\hline Vec. Propio 6 & & $\begin{array}{c}0,11816 \\
(0,03262)\end{array}$ & \\
\hline Vec. Propio 4 & & $\begin{array}{l}-0,12672 \\
(0,03447)\end{array}$ & \\
\hline Vec. Propio 2 & & $\begin{array}{c}0,11593 \\
(0,03327) \\
\end{array}$ & \\
\hline Vec. Propio 28 & & $\begin{array}{l}-0,10626 \\
(0,03354)\end{array}$ & \\
\hline Vec. Propio 29 & & $\begin{array}{c}0,08457 \\
(0,03278)\end{array}$ & \\
\hline Vec. Propio 31 & & $\begin{array}{c}0,07799 \\
(0,03304)\end{array}$ & \\
\hline Vec. Propio 10 & & $\begin{array}{c}0,06993 \\
(0,03350)\end{array}$ & \\
\hline Vec. Propio 15 & & $\begin{array}{c}0,06609 \\
(0,03313)\end{array}$ & \\
\hline Vec. Propio 30 & & $\begin{array}{c}0,06161 \\
(0,03296)\end{array}$ & \\
\hline Vec. Propio 26 & & $\begin{array}{l}0,05558 \\
(0,03281)\end{array}$ & \\
\hline Coef. de convergencia $\lambda$ & 0,07107 & 0,07603 & 0,60610 \\
\hline RSS & 0,0372 & 0,0325 & 0,02634 \\
\hline $\mathrm{R}^{2}$ (adj.) & $\begin{array}{c}0,354 \\
(0,342)\end{array}$ & $\begin{array}{c}0,529 \\
(0,496)\end{array}$ & $\begin{array}{c}0,724 \\
(0,669)\end{array}$ \\
\hline Criterio de información de Akaike & $-794,8351$ & $-842,4925$ & $-914,6238$ \\
\hline Índice de Moran & $0,09646^{* \star \star}$ & $-0,05453$ & $-0,05572$ \\
\hline Test de Breusch-Pagan & $\begin{array}{l}9,729^{* *} \\
(\mathrm{df}=4)\end{array}$ & $\begin{array}{l}23,904^{* *} \\
(\mathrm{df}=14)\end{array}$ & $\begin{array}{l}21,238 \\
(\mathrm{df}=35)\end{array}$ \\
\hline Test de Jarque-Bera & $\begin{array}{l}8,0539^{* *} \\
(\mathrm{df}=2)\end{array}$ & $\begin{array}{c}4,0905 \\
(\mathrm{df}=2)\end{array}$ & $\begin{array}{l}1,0163 \\
(\mathrm{df}=2)\end{array}$ \\
\hline Error cuadrático medio (RMSE) & 0,03673 & 0,03136 & 0,02402 \\
\hline
\end{tabular}

Fuente: Elaboración propia.

Nota: $\quad{ }^{* \star *}$ significativo al 1\%; ${ }^{* \star}$ significativo al 5\%; ${ }^{*}$ significativo al 10\%. Errores estándar entre paréntesis.

El coeficiente de determinación $R^{2}$ se incrementa considerablemente (de 0,35 a 0,72) en el caso de la filtración espacial-GWR, mientras que en el caso de la filtración espacial con interceptos individuales es de 0,53. El criterio de información de Akaike confirma este resultado, al igual que el error cuadrático medio (RMSE), que se utiliza para medir las diferencias entre los valores predichos por un modelo y los valores realmente observados, que mejora de manera notable en el modelo 
FS-GWR. Al mismo tiempo, se observa que en el segundo y el tercer modelo no existe autocorrelación espacial entre los residuos, dado que el test de Moran no es estadísticamente significativo. La heterocedasticidad persiste en los primeros dos modelos, mientras el test de Breusch-Pagan no es significativo en el caso de la filtración espacial-GWR. El coeficiente asociado al logaritmo del VAB per cápita en 2007 disminuye de manera marginal en el último modelo. La educación y el capital físico son significativos en todos los casos, lo que confirma su papel como motor del crecimiento, tal como se ha observado en otros países latinoamericanos. El papel de la educación en el crecimiento del Ecuador confirma lo encontrado por Szeles y Mendieta Muñoz (2016) con un modelo de datos de panel para las provincias ecuatorianas entre 2007 y 2014. La importancia del capital humano en el crecimiento también se ha demostrado en otros países sudamericanos, como el Brasil (Cravo, Becker y Gourlay, 2015; Özyurt y Daumal, 2013; De Andrade Lima y Silveira Neto, 2016) y México (RodríguezPose y Villarreal, 2015).

En el cuadro 2 se testean los tres modelos, uno contra otro, con la prueba ANOVA. Al analizar el valor $p$ de la prueba de chi cuadrado, la conclusión es que ambos modelos espaciales mejoraron significativamente con respecto al modelo de mínimos cuadrados ordinarios. En la comparación entre el modelo estimado con filtración espacial y el modelo con filtración espacial-GWR se observa que el último tiene un mejor rendimiento que los dos modelos de base.

Cuadro 2

Prueba ANOVA

\begin{tabular}{lcc}
\hline & $\begin{array}{c}\text { Desviación } \\
\text { suma de cuadrado }\end{array}$ & df \\
\hline Mínimos cuadrados ordinarios vs. Filtros espaciales & $0,1652^{\star \star \star}$ & 31 \\
\hline Mínimos cuadrados ordinarios vs. Filtros espaciales GWR & $0,0782^{\star \star \star}$ & 10 \\
\hline Filtros espaciales vs. Filtros espaciales GWR & $0,0870^{\star \star \star}$ & 21 \\
\hline
\end{tabular}

Fuente: Elaboración propia.

Nota: $\quad$ *** significativo al $1 \%$; ${ }^{* \star}$ significativo al $5 \%$; ${ }^{*}$ significativo al $10 \%$.

De acuerdo con Brunsdon, Fotheringham y Charlton (1998 y 1999), y Fotheringham, Brunsdon y Charlton (2002, pág. 229), es posible averiguar la no estacionariedad de los coeficientes GWR con dos procedimientos que se pueden extender sencillamente al caso de filtración espacial-GWR (véase el cuadro 3).

Cuadro 3

Test para no estacionariedad de los parámetros

\begin{tabular}{lcccccc}
\hline Variable & $\begin{array}{c}\text { Desv. est. } \\
\beta_{F S-G W R}\end{array}$ & $\operatorname{SE}\left(\beta_{\text {OLS }}\right)$ & $\operatorname{SE}\left(\beta_{F S}\right)$ & $\begin{array}{c}\text { Rango intercuar. } \\
\beta_{F S-G W R}\end{array}$ & $2 \times \operatorname{SE}\left(\beta_{\text {OLS }}\right)$ & $2 \times \operatorname{SE}\left(\beta_{F S}\right)$ \\
\hline Intercepto & 0,3331 & 0,0631 & 0,0642 & 0,2720 & 0,1261 & 0,1284 \\
\hline Log $(\mathrm{VAB} /$ pob $)$ & 0,0526 & 0,0062 & 0,0055 & 0,0436 & 0,0123 & 0,0111 \\
\hline Crec. pobl. & 0,0895 & 0,0105 & 0,0142 & 0,0864 & 0,0210 & 0,0285 \\
\hline Educación & 0,0947 & 0,0171 & 0,0161 & 0,0867 & 0,0342 & 0,0321 \\
\hline Capital & 0,0025 & 0,0005 & 0,0004 & 0,0020 & 0,0009 & 0,0008 \\
\hline
\end{tabular}

Fuente: Elaboración propia.

El primer paso es comparar la varianza de los coeficientes GWR con los errores estándar de los coeficientes estimados con mínimos cuadrados ordinarios. En el cuadro 4, todos los valores de varianza de los coeficientes FS-GWR (segunda columna) exceden los errores estándar de los coeficientes estimados con modelos estacionarios, mínimos cuadrados ordinarios y FS (tercera y cuarta columnas). Esto sugiere que hay una justificación para considerar coeficientes que varían 
en el espacio para todos los coeficientes. En segundo lugar, hay que comparar la diferencia entre el cuartil inferior y superior de los coeficientes locales (quinta columna) con respecto a dos veces las desviaciones estándar de la respectiva estimación global (sexta y séptima columnas), es decir \pm 1 desviación estándar de la media. Dado que se espera que el 68\% de los valores se encuentren dentro de este último intervalo, comparado con el 50\% en el rango intercuartil, y dado que el rango intercuartílico de los coeficientes locales es mayor que el de 2 errores estándar de la media global, esto sugiere que la relación es no estacionaria.

Cuadro 4

Valores locales de los coeficientes significativos

\begin{tabular}{lcccccccc}
\hline Variable & Mínimo & Primer cuartil & Media & Tercer cuartil & Máximo & $\begin{array}{c}\text { Porcentaje } \\
\text { de cantidad } \\
\text { significativa }\end{array}$ & $\begin{array}{c}\text { Porcentaje de } \\
\text { cantidad con } \\
\text { valores }>0\end{array}$ & $\begin{array}{c}\text { Índice de } \\
\text { Moran }\end{array}$ \\
\hline Intercepto & $-1,7952$ & $-0,3195$ & $-0,0843$ & 0,2395 & 0,9934 & 0,528 & 0,655 & $0,518^{\star * \star}$ \\
\hline Tasa de conv. $\lambda_{i}$ & 0,0027 & 0,0539 & 0,0964 & 0,1191 & 0,6718 & 0,977 & 0,096 & $0,474^{\star * *}$ \\
\hline Crecimiento pobl. & $-0,3810$ & $-0,0949$ & $-0,0408$ & $-0,0320$ & 0,3487 & 0,692 & 0,784 & $0,478^{* \star *}$ \\
\hline Educación & $-0,1762$ & 0,1119 & 0,1602 & 0,190 & 0,4390 & 0,930 & 0,020 & $0,650^{\star * *}$ \\
\hline Capital & $-0,1762$ & 0,1132 & 0,1593 & 0,1908 & 0,4390 & 0,771 & 0,036 & $0,432^{\star * *}$ \\
\hline
\end{tabular}

Fuente: Elaboración propia

Nota: ${ }^{\star \star \star}$ significativo al $1 \%$, ${ }^{* \star}$ significativo al $5 \%$ y ${ }^{\star}$ significativo al $10 \%$. Significatividad de la I de Moran basada en 1.000 aleatorizaciones.

En el cuadro 4 se encuentran los valores locales de los coeficientes. Para averiguar si son estadísticamente diferentes de cero se calculan los errores estándar tomando en cuenta que estos se derivan a partir de los términos de interacción (Dawson y Richter, 2006). Se observa que el valor medio (global) no coincide exactamente con el valor del coeficiente estimado para las variables en el cuadro 1, puesto que en el cuadro 4 se consideran solo las variables locales estadísticamente diferentes de cero. Para todas las variables existe una marcada brecha entre el primer y el tercer cuartil, lo que revela un alto grado de variabilidad. Además, el 98\% de los cantones muestran un coeficiente significativo y alrededor del $10 \%$ diverge. Con respecto a las otras variables incluidas en el modelo, la tasa de crecimiento de la población es significativa en el $69 \%$ de los cantones. Sorprendentemente, la educación y el capital físico son significativos en un 93\% y un $77 \%$ de los cantones, respectivamente, lo que indica que existen áreas donde estas variables no afectan al crecimiento. Esto probablemente es producto de la heterogeneidad estructural que se refleja en una concentración de los sectores productivos en pocas áreas; por lo general alrededor de las ciudades principales (Mendieta Muñoz y Pontarollo, 2016). La última columna representa el Índice de Moran de los coeficientes locales asociados a cada variable. Todos los IM son positivos y significativos, lo que determina un patrón espacial bien definido, con cantones caracterizados por valores próximos situados cerca en el espacio.

Lo expuesto resulta evidente en el cuadro 5 , donde se presentan los vectores propios asociados a cada variable, junto con su escala geográfica. Estos valores se utilizan principalmente para medir el grado de heterogeneidad u homogeneidad espacial. De hecho, dado que, como se explicó en la sección III, cada vector propio tiene un IM asociado, si la escala prevaleciente de los vectores propios asociados a una variable es la local, esto significa que existe una fuerte heterogeneidad, es decir, que el fenómeno objeto de estudio tiene un impacto sobre la variable dependiente que varía significativamente entre un territorio y otro. Por el contrario, si la escala prevaleciente es la global, ello significa que áreas homogéneas impactan de forma similar con respecto a la variable dependiente. En el caso de que una variable no presente vectores propios asociados, ello implica que esta variable no tiene impactos diferenciados en el espacio, es decir, su impacto para cada localización 
es el mismo. En el caso puntual que se está analizando, todas las variables tienen vectores propios asociados con escala prevalecientemente local y regional, lo que confirma la existencia de clústeres bien definidos, sobre todo en las zonas de la sierra, como lo muestra el Índice de Moran local en los mapas del anexo $\mathrm{A}^{8}$.

Cuadro 5

Valores propios asociados a cada variable

\begin{tabular}{lccc}
\hline & $\begin{array}{c}\text { Escala local } \\
(0.50>I M>0.25)\end{array}$ & $\begin{array}{c}\text { Escala regional } \\
(0.75>I M>0.50)\end{array}$ & $\begin{array}{c}\text { Escala global } \\
(I M>0.75)\end{array}$ \\
\hline Intercepto & 3 & 1 & 1 \\
\hline Log (VAB per cápita 2007) & 2 & 3 & 1 \\
\hline Crecimiento pobl. & 3 & 3 & 0 \\
\hline Educación & 3 & 2 & 1 \\
\hline Capital & 7 & 1 & 0 \\
\hline
\end{tabular}

Fuente: Elaboración propia.

En el mapa 2 se muestra la variación local del coeficiente asociado al intercepto. Los cantones donde los factores estructurales considerados por el valor local del intercepto tienen el mayor impacto se encuentran ubicados al sur del país, así como, entre la región costa y sierra, en la parte septentrional del Ecuador. Los clústeres de valores bajos están en las provincias de Guayas y El Oro.

Mapa 2

Ecuador: coeficientes locales del intercepto
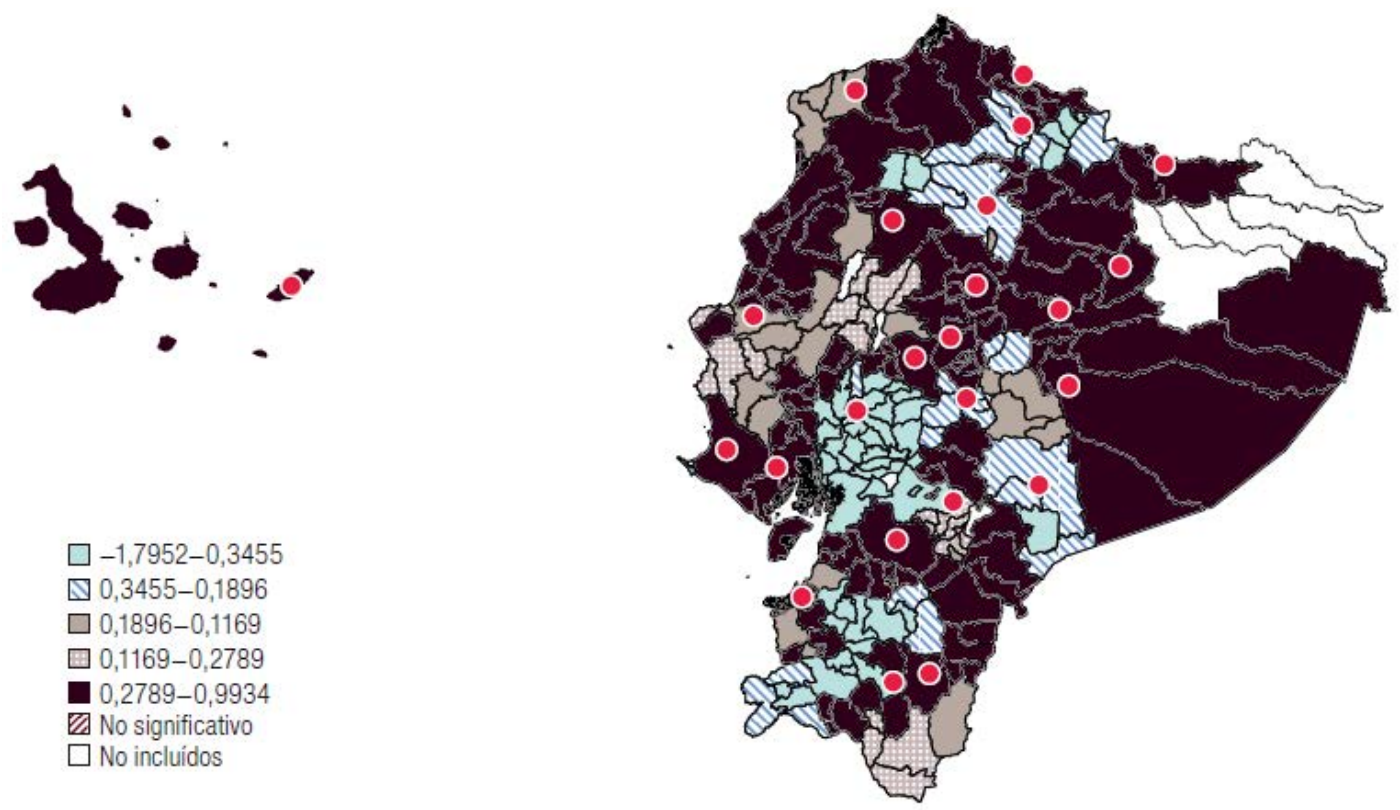

Fuente: Elaboración propia.

Nota: Los puntos representan las capitales provinciales.

\footnotetext{
8 Véase la distribución de las provincias y zonas en el anexo A1.
} 
En el mapa 3 se presenta la variación local de la tasa de convergencia. Las zonas donde se registra la mayor velocidad de convergencia se encuentran ubicadas en el hot spot de cantones que pertenecen a las provincias ubicadas al sur de Quito, en la parte meridional del país, y en la costa, con excepción de la zona centro-septentrional. Por último, las tasas de convergencia con menor valor se presentan en el centro de la sierra. Además, se observan ciertos cantones que divergen (en rojo), en la provincia de Cañar y en el norte del país, donde también se ubican los clústeres de cantones con baja tasa de convergencia.

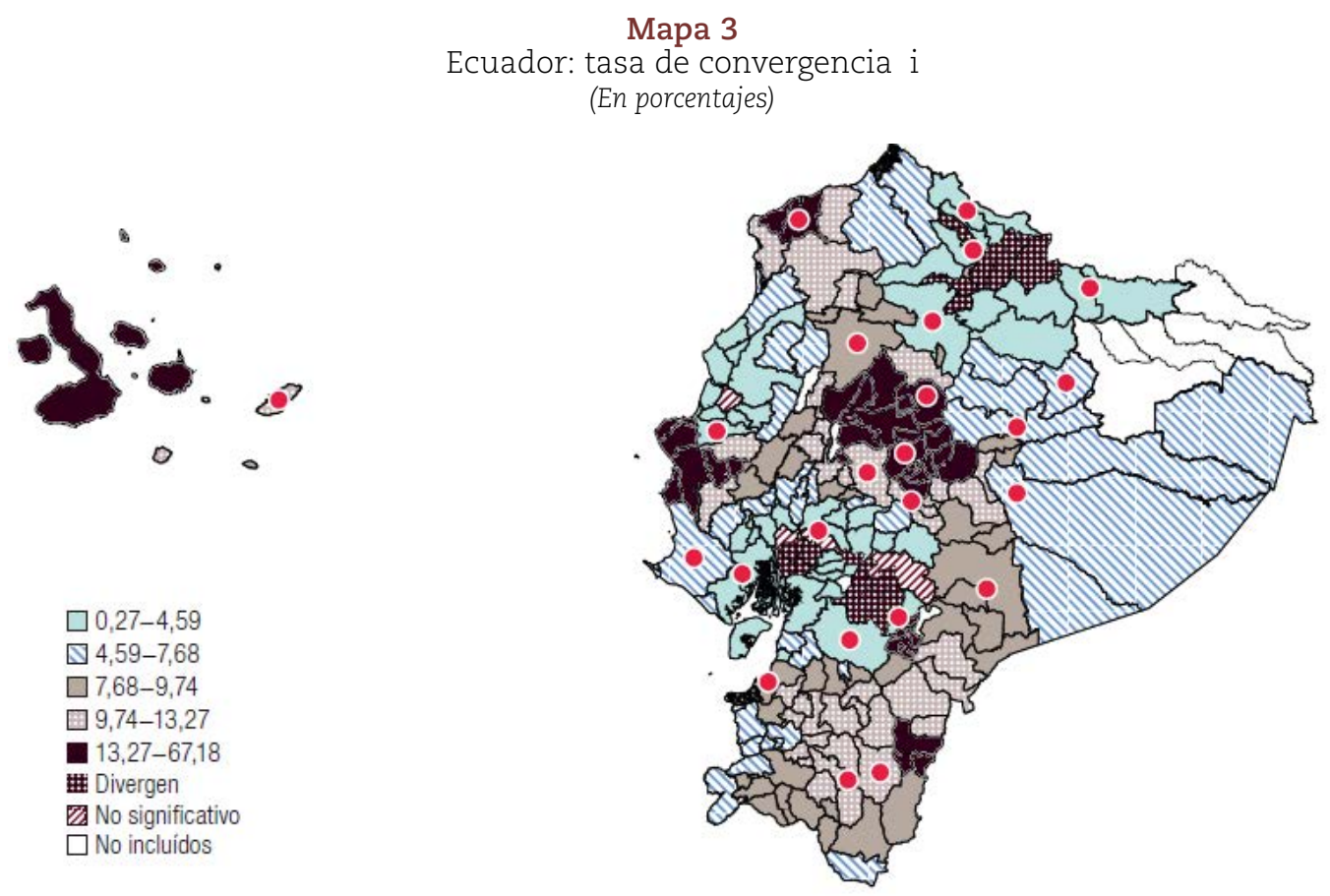

Fuente: Elaboración propia.

Nota: Los puntos representan las capitales provinciales.

En los mapas 4 y 5 se muestran, respectivamente, los coeficientes de $\ln \left(n_{i}+g+\delta\right)$ y del capital físico. Lo primero que se observa es que existen varios cantones donde los coeficientes no son significativos. Con respecto al crecimiento de la población, estos se ubican generalmente en la parte central de la costa, en el límite con el Perú y con Colombia. El capital físico no es significativo en la parte norte de la costa, en la Amazonia ni en la sierra, en la provincia de Cañar.

En relación con las áreas donde existe significancia estadística de la tasa de crecimiento de la población, los coeficientes más elevados se encuentran ubicados en la zona noroeste. Con respecto al capital, el impacto es mayor en los cantones de frontera entre la sierra y la costa. 


\section{Mapa 4}

Ecuador: coeficientes locales de la tasa de crecimiento de la población
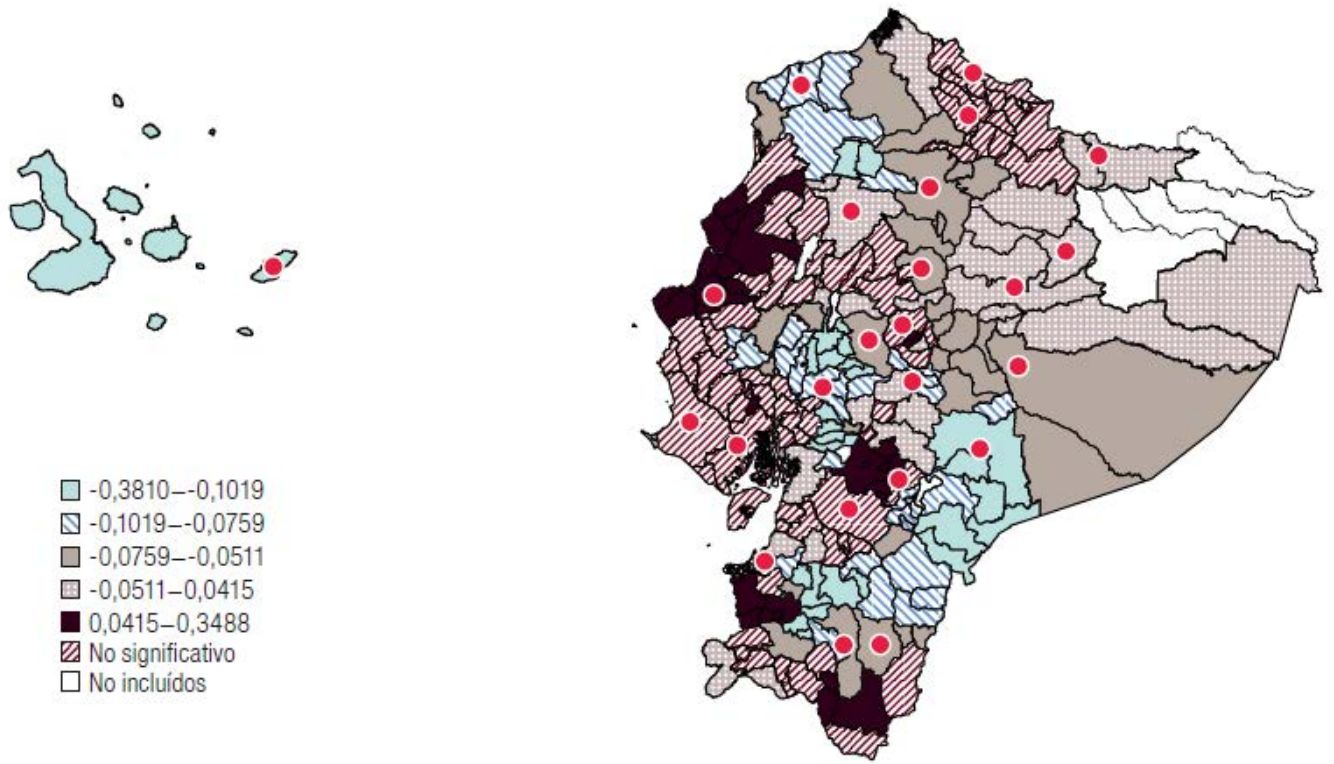

Fuente: Elaboración propia.

Nota: Los puntos representan las capitales provinciales.

Mapa 5

Ecuador: coeficientes locales del capital
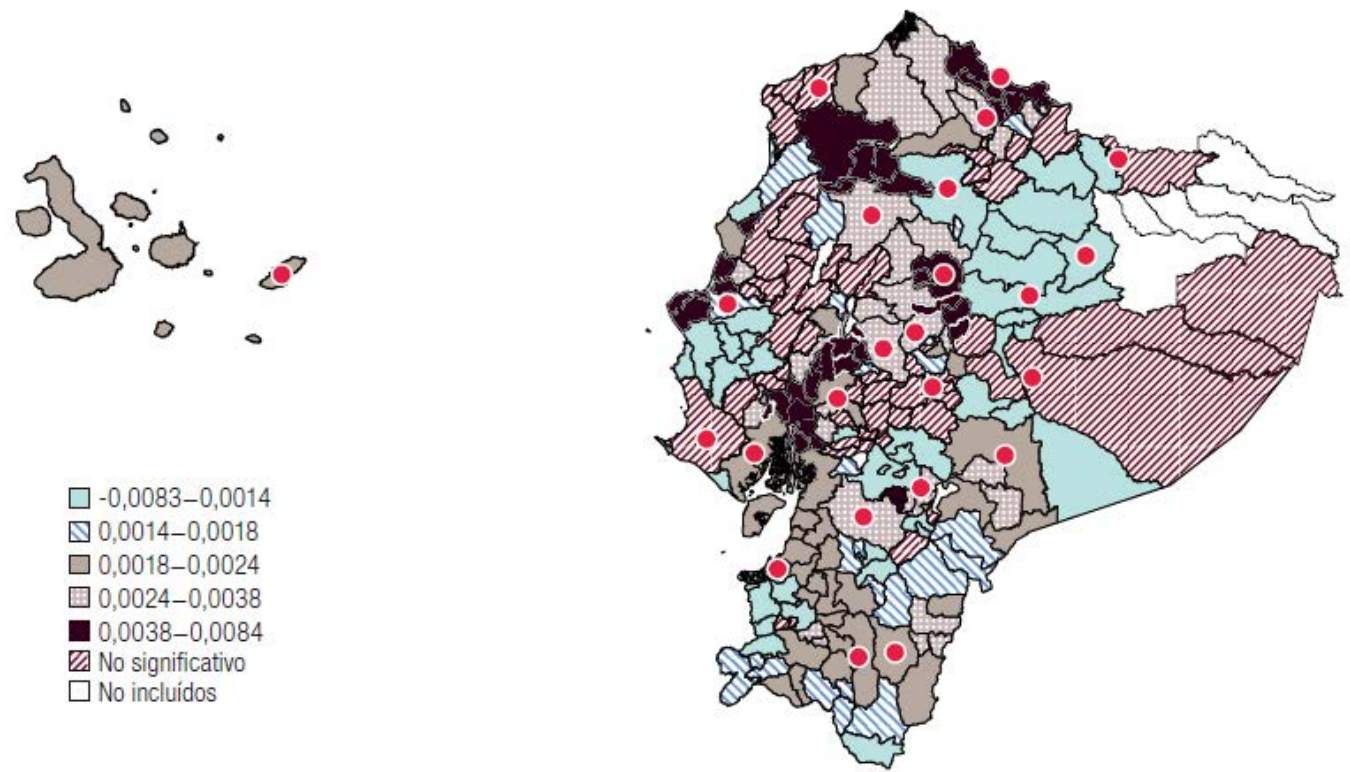

Fuente: Elaboración propia.

Nota: Los puntos representan las capitales provinciales.

Por último, en el mapa 6 se muestra cómo el mayor impacto de la educación se observa en la zona meridional del país y en el centro de la zona de la sierra, donde hay hot spots estadísticamente significativos de cantones. Además, se observan clústeres con un impacto muy bajo al norte de Quito y en Cañar y Azuay. 


\section{Mapa 6}

Ecuador: coeficientes locales de la educación
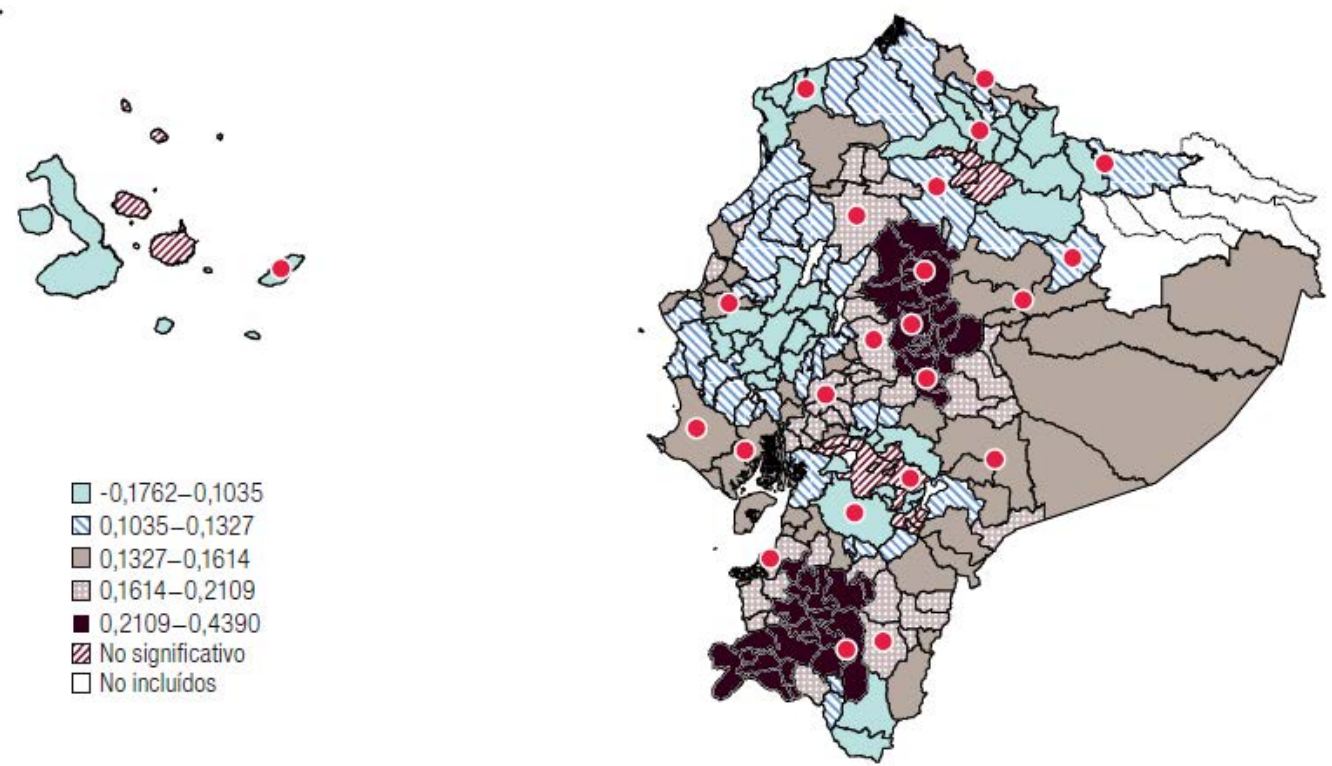

Fuente: Elaboración propia.

Nota: Los puntos representan las capitales provinciales.

La correlación entre el coeficiente del intercepto y la velocidad de convergencia $\beta$ es de 0.472 , lo que indica que existe una relación directa, pero moderada, entre los factores estructurales no observados que afectan el crecimiento y la tasa de convergencia cantonal (véase el cuadro 6). En este sentido, alrededor de la mitad de los cantones que registran elevados ritmos de convergencia también disfrutan de ciertas condiciones estructurales que promueven e impulsan su crecimiento y viceversa. La correlación entre el término del intercepto y los coeficientes del capital humano y físico es negativa y muy baja. En otras palabras, el impacto de la educación y del capital físico sobre el crecimiento económico cantonal es mayor en los cantones donde las condiciones estructurales son más adversas y viceversa. Esto da cuenta de que el retorno a la inversión en capital humano y físico es menor en aquellos cantones que han alcanzado cierto grado de desarrollo.

Cuadro 6

Correlación entre los valores locales de los parámetros

\begin{tabular}{lccccc}
\hline & Intercepto & $\lambda_{i}$ & Crec. pobl. & Educación & Capital \\
\hline Intercepto & & & & & \\
\hline Tasa de conv. $\lambda_{i}$ & 0,472 & & & & \\
\hline Crecimiento pobl. & 0,175 & $-0,104$ & & & \\
\hline Educación & $-0,258$ & 0,267 & $-0,099$ & & \\
\hline Capital & $-0,260$ & 0,257 & $-0,089$ & 0,813 & 1
\end{tabular}

Fuente: Elaboración propia.

Por otra parte, la correlación entre el impacto del capital humano y físico muestra un coeficiente positivo de 0.813 , lo que significa que estos dos factores se complementan. A pesar de este resultado, se debe señalar que existen ciertas zonas del país, en especial la sierra central y la Amazonia, que presentan un efecto de compensación entre el capital físico y humano: cuanto mayor es el impacto del 
capital físico, menor es el del capital humano. Esto puede obedecer a las características económicas de estos cantones, que hacen uso de capital físico y humano con mayor o menor intensidad, dependiendo de su especialización productiva.

\section{Conclusiones}

En este trabajo se analiza el proceso de convergencia condicional en el Ecuador entre 2007 y 2015 a través de un modelo econométrico espacial que toma en cuenta la heterogeneidad estructural de los cantones y la autocorrelación espacial. Si bien el modelo utilizado es una evolución de la técnica GWR (Fotheringham, Brunsdon y Charlton, 2002), la técnica FS-GWR no impone que los coeficientes sean no estacionarios, pero lo admite, lo que significa que en la misma regresión puede haber coeficientes globales y locales, como habría en una GWR mixta, pero resolviendo los problemas de multicolinealidad y de falta de grados de libertad. Para evaluar la convergencia se utiliza el VAB per cápita que, como se demuestra en este estudio, incrementa su varianza en conjunto con la autocorrelación espacial, lo que señala una creciente disparidad y polarización económica intercantonal.

Este análisis se distingue de otros aportes anteriores (Mendieta Muñoz, 2015; RamónMendieta, Ochoa-Moreno y Ochoa-Jiménez, 2013; Mendieta Muñoz y Pontarollo, 2016; Szeles y Mendieta Muñoz, 2016) en que estima el modelo de convergencia $\beta$ condicional haciendo uso de los filtros espaciales, una técnica econométrica que permite tener en cuenta la inestabilidad de los parámetros. Por lo tanto, no es necesario asumir, como se hizo en estudios previos, que todos los cantones responden de manera semejante ante los factores que determinan el crecimiento, sino que se admite la posibilidad de que las variables incluidas en el modelo tengan un efecto diferenciado en el espacio, en función de las peculiaridades de cada territorio.

En este sentido, se demuestra que el impacto de la educación se concentra en la zona central de la sierra, donde hay una notoria aglomeración de ciudades, y cerca de los límites con el Perú, que son las áreas más dinámicas, donde hay más flujos de personas y mercancías. Al revés, en la zona central de la costa, al norte de Guayaquil, en Cañar, y en la parte norte del país la educación tiene un papel limitado o nulo con respecto al crecimiento. Esto puede responder al hecho de que los cantones que se encuentran cerca de las mayores ciudades (Guayaquil, Quito y Cuenca) parecen verse perjudicados por estas urbes porque, de acuerdo con Mendieta Muñoz y Pontarollo (2016), ellas absorben los recursos de las áreas cercanas. El impacto positivo del capital físico, por otra parte, se sobrepone geográficamente al efecto del capital humano.

Por último, en el contexto de la convergencia condicional, la presencia de un alto grado de heterogeneidad de los coeficientes de convergencia muestra que hay algunos catones que están próximos a su propio estado estacionario.

La inestabilidad en los parámetros asociada a la dimensión espacial da cuenta de que en el Ecuador no solo existen evidentes desigualdades entre un territorio y otro, sino que esto implica marcadas asimetrías en los efectos de los factores que condicionan la convergencia. Estas diferencias responderían a la presencia de un componente de contagio espacial. Así, el análisis de los factores que afectan la convergencia en el caso ecuatoriano toma una connotación completamente distinta y se debe tener absoluto cuidado al hacer un análisis general del país.

Con esto, sostenemos que, desde el punto de vista de la política económica, se debe poner énfasis en que el hecho de que los cantones tengan características estructurales diferentes implica que las políticas económicas también sean distintas, debido a que las regiones no son países y no pueden simplemente replicar las políticas nacionales a nivel regional (OCDE, 2011, pág. 19). 
Esto se relaciona con la evidencia de que, como han destacado varios autores (véase, por ejemplo, Barca, McCann y Rodríguez-Pose, 2012), para explotar el potencial de crecimiento de cada territorio es esencial lograr una comprensión detallada de su estructura socioeconómica con el fin de adaptar la política pública local a sus necesidades particulares, encontrando la combinación adecuada de intervenciones.

En este sentido, la herramienta propuesta, que permite comprender las dinámicas territoriales de manera más profunda, puede servir para construir políticas adecuadas al contexto territorial y, por lo tanto, más eficaces. En el caso ecuatoriano, caracterizado por marcadas diferencias estructurales, las políticas incorrectas pueden perjudicar el desarrollo balanceado del país y llevar a un fenómeno de convergencia hacia abajo (Szeles y Mendieta Muñoz, 2016), donde los cantones más desarrollados decrecen hacia el nivel de los menos desarrollados, y no al revés.

\section{Bibliografía}

Abreu, M., H. L. de Groot y R. J. Florax (2005), "A meta-analysis of $\beta$-convergence: the legendary 2\%", Journal of Economic Surveys, vol. 19, № 3, Hoboken, Wiley.

Anselin, L. (1995), "Local indicators of spatial association - LISA", Geographical Analysis, vol. 27, № 2, Hoboken, Wiley.

(1988), Spatial Econometrics: Methods and Models, Dordrecht, Kluwer Academic Publishers.

Arbia, G. (2006), Spatial Econometrics: Statistical Foundations and Applications to Regional Convergence, Berlín, Springer.

Arbia, G., M. Battisti y G. di Vaio (2010), "Institutions and geography: empirical test of spatial growth models for European regions", Economic Modelling, vol. 27, N 1, Amsterdam, Elsevier.

Azzoni, C. (2001), "Economic growth and regional income inequality in Brazil", The Annals of Regional Science, vol. 35, No 1, Berlín, Springer.

Barca, F., P. McCann y A. Rodríguez-Pose (2012), "The case for regional development intervention: placebased versus place-neutral approaches", Journal of Regional Science, vol. 52, № 1, Hoboken, Wiley.

Barro, R. J. y J. Lee (1993), "International comparisons of educational attainment", Journal of Monetary Economics, vol. 32, $\mathrm{N}^{\circ} 3$, Amsterdam, Elsevier.

Barro, R. J. y X. Sala-i-Martin (1992), "Convergence”, Journal of Political Economy, vol. 100, № 2, Chicago, The University of Chicago Press.

(1991), "Convergence across states and regions", Brookings Papers on Economic Activity, vol. 22, № 1, Washington, D.C., Brookings Institution.

Baumol, W. J. (1986), "Productivity growth, convergence, and welfare: what the long-run data show", The American Economic Review, vol. 76, № 5, Nashville, Tennessee, American Economic Association.

Benhabib, J. y M. Spiegel (1994), "The role of human capital in economic development evidence from aggregate cross-country data", Journal of Monetary Economics, vol. 34, Nㅜ 2, Amsterdam, Elsevier.

Bockstael, N. E. (1996), "Modeling economics and ecology: the importance of a spatial perspective", American Journal of Agricultural Economics, vol. 78, № 5, Oxford, Oxford University Press.

Brunsdon C., A. S. Fotheringham y M. Charlton (1999), "Some notes on parametric significance tests for geographically weighted regression", Journal of Regional Science, vol. 39, № 3, Hoboken, Wiley.

(1998), "Geographically weighted regression-modeling spatial non-stationarity", The Statistician, vol. 47, № 3, Hoboken, Wiley.

Canarella, G. y S. Pollard (2006), "Distribution dynamics and convergence in Latin America: a non-parametric analysis", International Review of Economics, vol. 53, № 1, Berlín, Springer.

Cárdenas, M. y A. Ponton (1995), "Growth and convergence in Colombia: 1950-1990", Journal of Development Economics, vol. 47, № 1, Amsterdam, Elsevier.

Celebioglu, F. y S. Dall'erba (2010), "Spatial disparities across the regions of Turkey: an exploratory spatial data analysis", The Annals of Regional Science, vol. 45, №2, Berlín, Springer.

Cravo, T., B. Becker y A. Gourlay (2015), "Regional growth and SMEs in Brazil: a spatial panel approach", Regional Studies, vol. 49, No 12, Abingdon, Taylor \& Francis.

Cravo, T. y G. M. Resende (2013), "Economic growth in Brazil: a spatial filtering approach", The Annals of Regional Science, vol. 50, №2, Berlín, Springer. 
Cuadrado-Roura, J., T. Mancha-Navarro y R. Garrido-Yserte (2000), "Regional productivity patterns in Europe: an alternative approach", The Annals of Regional Science, vol. 34, № 3, Berlín, Springer.

Dawson, J. F. y A. W. Richter (2006), "Probing three-way interactions in moderated multiple regression: development and application of a slope difference test", Journal of Applied Psychology, vol. 91, $\mathrm{N}^{\circ} 4$, Washington, D.C., American Psychological Association.

De Andrade Lima, R. y R. Silveira Neto (2016), "Physical and human capital and Brazilian regional growth: a spatial econometric approach for the period 1970-2010", Regional Studies, vol. 50, № 10, Abingdon, Taylor \& Francis.

De Dominicis, L. (2014), "Inequality and growth in European regions: towards a place-based approach", Spatial Economic Analysis, vol. 9, № 2, Abingdon, Taylor \& Francis.

De la Fuente, A. (1994), "Crecimiento y convergencia: un panorama selectivo de la evidencia empírica", Cuadernos Económicos de ICE, N 58 , Madrid, Ministerio de Economía, Industria y Competitividad.

Durlauf, S. N., P. A. Johnson y J. Temple (2005), "Growth econometrics", Handbook of Economic Growth, P. Aghion y S. N. Durlauf (eds.), Amsterdam, North-Holland.

Ertur, C., J. Le Gallo y J. P. LeSage (2007), "Local versus global convergence in Europe: a Bayesian spatial econometric approach", The Review of Regional Studies, vol. 37, Nㅜ 1, Southern Regional Science Association.

Esquivel, G. (1999), “Convergencia regional en México, 1940-1995”, Trimestre Económico, vol. 66, № 264 , Ciudad de México, Fondo de Cultura Económica.

Fingleton, B. (1999), "Estimates of time to economic convergence: an analysis of regions of the European Union", International Regional Science Review, vol. 22, № 1, Thousand Oaks, Sage.

Fischer, M. y D. Griffith (2008), "Modeling spatial autocorrelation in spatial interaction data: an application to patent citation data in the European Union", Journal of Regional Science, vol. 48, № 5, Hoboken, Wiley.

Fotheringham, A. S., C. Brunsdon y M. Charlton (2002), Geographically Weighted Regression: The Analysis of Spatially Varying Relationships, Chichester, Wiley.

Fotopoulos, G. (2012), "Nonlinearities in regional economic growth and convergence: the role of entrepreneurship in the European union regions", The Annals of Regional Science, vol. 48, № 3, Berlín, Springer .

Fujita M., P. Krugman y A. J. Venables (1999), The Spatial Economy, Cambridge, MIT Press.

Getis, A. (1995), "Spatial filtering in a regression framework: examples using data on urban crime, regional inequality and government expenditures", New Directions in Spatial Econometrics, L. Anselin y R. J. Florax (eds.), Berlín, Springer.

Getis, A. y D. Griffith (2002), "Comparative spatial filtering in regression analysis", Geographical Analysis, vol. 34, № 2, Hoboken, Wiley.

Gómez, F. y L. Santana (2016), "Convergencia interregional en Colombia 1990-2013: un enfoque sobre la dinámica espacial", Ensayos sobre Política Económica, vol. 34, № 80, Amsterdam, Elsevier.

Gómez-Zaldívar, M., D. Ventosa-Santaulària y F. Wallace (2012), "Appendix for the PPP hypothesis and structural breaks: the case of Mexico", MPRA Paper, № 41055, Munich, University Library of Munich.

Griffith, D. (2010), "Spatial filtering", Handbook of Applied Spatial Analysis: Software Tools, Methods and Applications, M. Fischer y A. Getis (eds.), Berlín, Springer.

(2008), "Spatial-filtering-based contribution to a critique of geographically weighted regression (GWR)", Environment and Planning A: Economy and Space, vol. 40, № 11, Thousand Oaks, Sage. (2003), Spatial Autocorrelation and Spatial Filtering: Gaining Understanding through Theory and Scientific Visualization, Berlín, Springer.

Islam, N. (2003), "What have we learnt from the convergence debate?", Journal of Economic Surveys, vol. 17, $N^{\circ} 3$, Hoboken, Wiley.

Ketteni, E., T. P. Mamuneas y T. Stengos (2007), "Nonlinearities in economic growth: a semiparametric approach applied to information technology data", Journal of Macroeconomics, vol. 29, № 3, Amsterdam, Elsevier.

Krugman, P. (1991), "Increasing returns and economic geography", Journal of Political Economy, vol. 99, $N^{\circ} 3$, Chicago, The University of Chicago Press.

Kyriacou, G. (1991), "Level and growth effects of human capital: a cross-country study of the convergence hypothesis", Working Paper, N 91-26, Nueva York, C.V. Starr Center for Applied Economics, New York University.

Lacombe, D. J. (2004), "Does econometric methodology matter? An analysis of public policy using spatial econometric techniques", Geographical Analysis, vol. 36, № 2, Hoboken, Wiley.

Le Gallo, J. y C. Ertur (2003), "Exploratory spatial data analysis of the distribution of regional per capita GDP in Europe, 1980-1995”, Papers in Regional Science, vol. 82, № 2, Hoboken, Wiley. 
LeSage, J. P. y M. Fischer (2008), "Spatial growth regressions: model specification, estimation and interpretation", Spatial Economic Analysis, vol. 3, №3, Abingdon, Taylor \& Francis.

Mankiw, N. G. (1995), "The growth of nations", Brookings Papers on Economic Activity, vol. 1, Washington, D.C., Brookings Institution.

Mankiw, N. G., D. Romer y D. N. Weil (1992), "A contribution to the empirics of economic growth", The Quarterly Journal of Economics, vol. 107, № 2, Oxford, Oxford University Press.

Mendieta Muñoz, R. (2015), "La hipótesis de la convergencia condicional en Ecuador: un análisis a nivel cantonal", Retos, vol. 5, № 9, Cuenca, Universidad Politécnica Salesiana.

Mendieta Muñoz, R. y N. Pontarollo (2016), "Cantonal convergence in Ecuador: a spatial econometric perspective", Journal of Applied Economic Sciences, vol. 39, № 11, Craiova, ASERS Publishing.

Moran, P. A. P. (1950), "Notes on continuous stochastic phenomena", Biometrika, vol. 37, № 1-2, Oxford, Oxford University Press.

Myrdal, G. (1957), Economic Theory and Under-developed Regions, Londres, Duckworth.

OCDE (Organización de Cooperación y Desarrollo Económicos) (2011), OECD Reviews of Regional Innovation: Regions and Innovation Policy, París, OECD Publishing.

Özyurt, S. y M. Daumal (2013), "Trade openness and regional income spillovers in Brazil: a spatial econometric approach", Papers in Regional Science, vol. 92, № 1, Hoboken, Wiley.

Pede, V. O., R. J. Florax y D. M. Lambert (2014), "Spatial econometric STAR models: Lagrange multiplier tests, Monte Carlo simulations and an empirical application", Regional Science and Urban Economics, vol. 49, Amsterdam, Elsevier.

Pina, Á. y M. S. Aubyn (2005), "Public capital, human capital and economic growth: Portugal, 1977-2001", Focus on Economic Growth and Productivity, L. A. Finley (ed.), Nueva York, Nova Science Publishers, Inc.

Postiglione P., M. S. Andreano y R. Benedetti (2017), "Spatial clusters in EU productivity growth", Growth and Change, vol. 48, No 1, Hoboken, Wiley.

Quah, D. (1996), "Regional convergence clusters across Europe", European Economic Review, vol. 40, $N^{\circ} 3-5$, Amsterdam, Elsevier.

Ramón-Mendieta, M., W. Ochoa-Moreno y D. Ochoa-Jiménez (2013), "Growth, clusters, and convergence in Ecuador: 1993-2011", Regional Problems and Policies in Latin America, J. Cuadrado-Roura y P. Aroca (eds.), Berlín, Springer.

Resende, G. M. y otros (2016), "Evaluating multiple spatial dimensions of economic growth in Brazil using spatial panel data models", The Annals of Regional Science, vol. 56, № 1, Berlín, Springer.

Rey, S. y B. Montouri (1999), "US regional income convergence: a spatial econometric perspective", Regional Studies, vol. 33, № 2, Abingdon, Taylor \& Francis.

Rodríguez-Pose, A. y E. Villarreal (2015), "Innovation and regional growth in Mexico: 2000-2010", Growth and Change, vol. 46, N ${ }^{\circ} 2$, Hoboken, Wiley.

Royuela, V. y G. García (2015), "Economic and social convergence in Columbia", Regional Studies, vol. 49, $N^{\circ}$ 2, Abingdon, Taylor \& Francis.

Sala-i-Martin, X. (1994), "La riqueza de las regiones. Evidencia y teorías sobre crecimiento regional y convergencia", Moneda y Crédito, № 198, Madrid, Fundación Banco Santander.

Serra, M. I. y otros (2006), "Regional convergence in Latin America", IMF Working Paper, No 06/125, Washington, D.C., Fondo Monetario Internacional (FMI).

Solow, R. (1999), "Neoclassical growth theory", Handbook of Macroeconomics, vol. 1, J. B. Taylor y M. Woodford (eds.), Amsterdam, North-Holland.

Szeles, M. y R. Mendieta Muñoz (2016), "Analyzing the regional economic convergence in Ecuador. Insights from parametric and nonparametric models", Romanian Journal of Economic Forecasting, vol. 19, Nㅜ 2, Bucarest, Institute for Economic Forecasting.

Temple, J. (2000), "Growth regressions and what the textbooks don't tell you", Bullettin of Economic Research, vol. 52, N³, Hoboken, Wiley.

(1998), "Robustness tests of the augmented Solow model", Journal of Applied Econometrics, vol. 13, № 4, Hoboken, Wiley.

Tiefelsdorf, M. y B. Boots (1995), "The exact distribution of Moran's I", Environment and Planning A: Economy and Space, vol. 27, $N^{\circ} 6$, Thousand Oaks, Sage.

Tobler, W. (1970), "A computer movie simulating urban growth in the Detroit region", Economic Geography, vol. 46, Abingdon, Taylor \& Francis.

Weinhold, D. (2002), "The importance of trade and geography in the pattern of spatial dependence of growth rates", Review of Development Economics, vol. 6, № 3, Hoboken, Wiley.

Wheeler, D. y M. Tiefelsdorf (2005), "Multicollinearity and correlation among local regression coefficients in geographically weighted regression”, Journal of Geographical Systems, vol. 7, № 2, Berlín, Springer. 


\section{Anexo A1}

$$
\text { Mapa A1.1 }
$$

Ecuador: mapa de las provincias
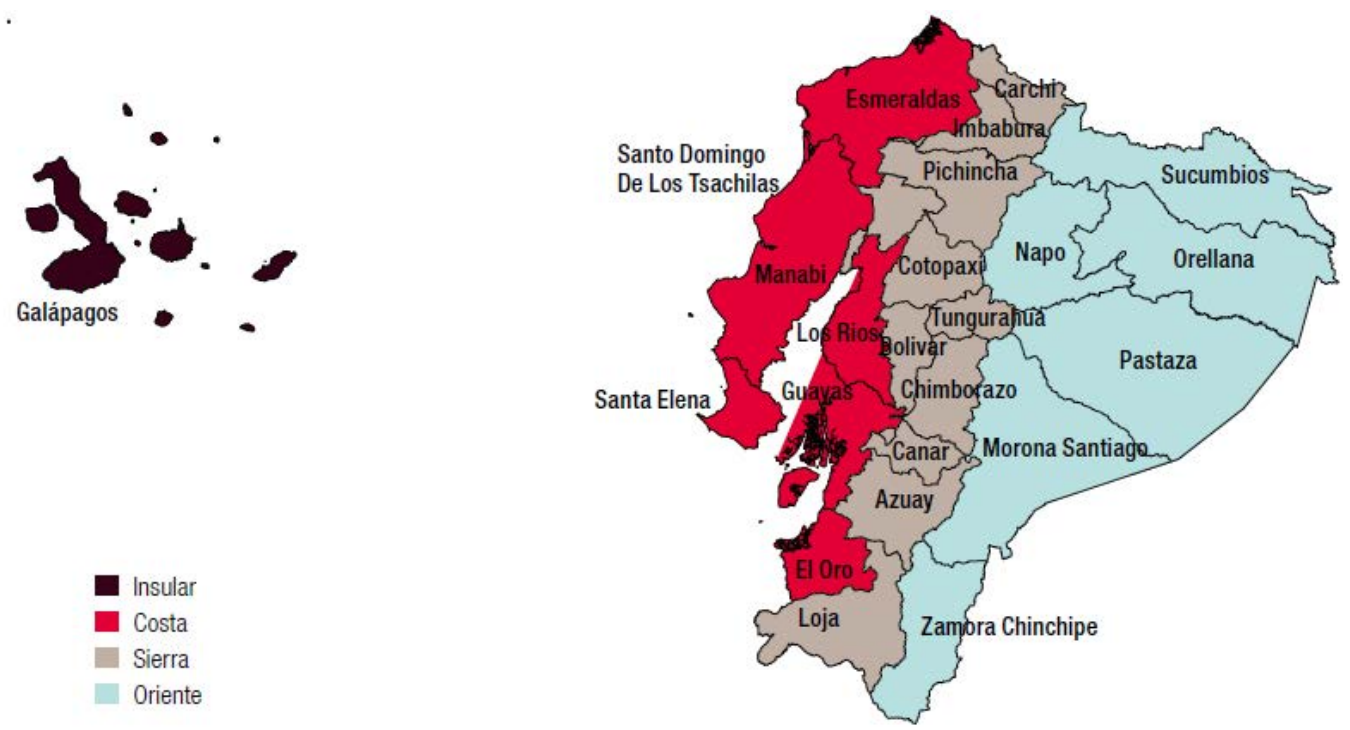

Fuente: Elaboración propia. 


\title{
Anexo A2
}

\author{
Mapa A2.1
}

Mapas de las variables del modelo

A. Crecimiento del VAB per cápita, 2007-2015

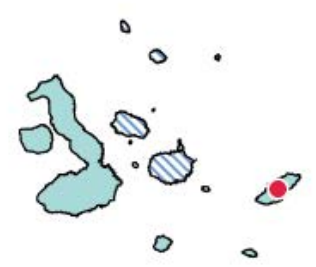

$-0,1757-0,0398$

$\triangle 0,0398-0,0126$

$\square 0,0126-0,0101$

$\square 0,0101-0,0384$

口 $0,0384-0,1517$

$\square$ No incluidos

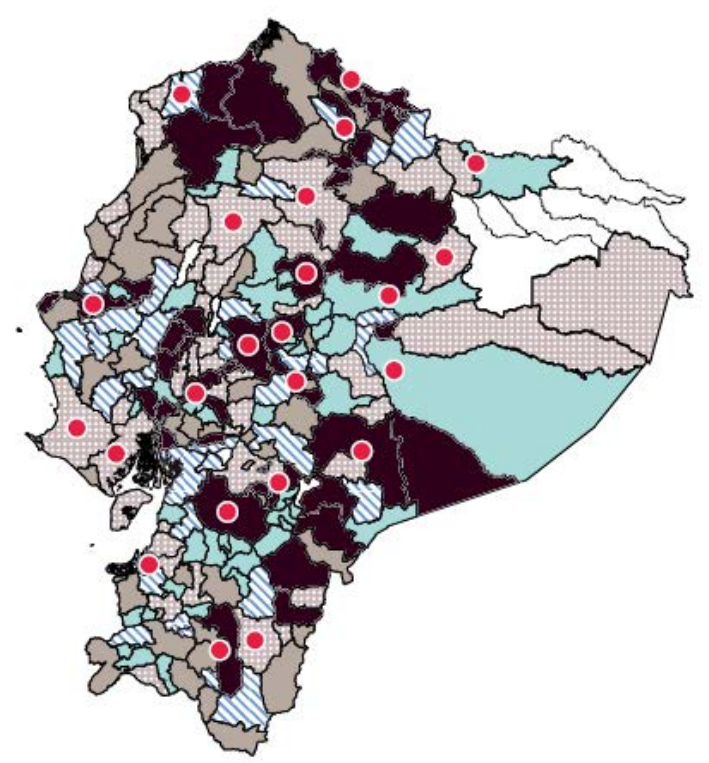

B. VAB per cápita, 2007

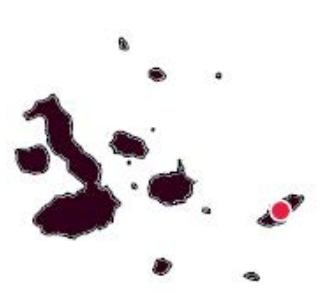

$\square 498,940-1313,812$

Q 313,812-1 621,090

$\square 1621,090-2013,054$

田 013,054-2 640,384

口 $2640,384-11324,790$

$\square$ No incluidos

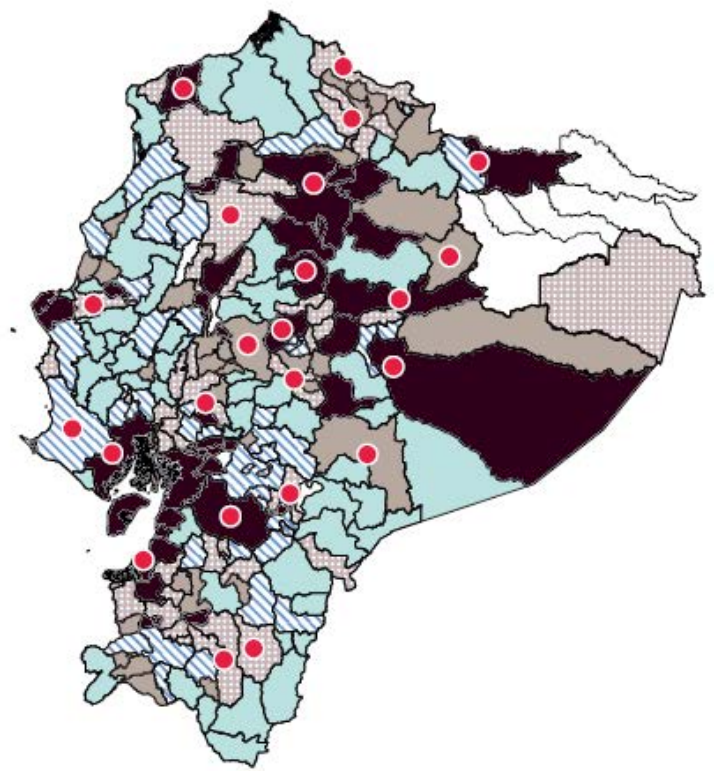


C. Crecimiento de la población, 2007-2015

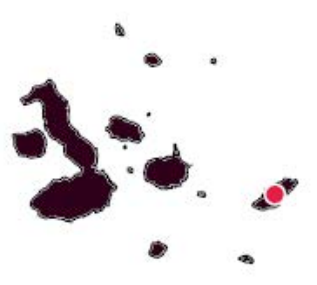

$\square-0,0119-0,0100$

Q $0,0100-0,0169$

$\square 0,0169-0,0220$

$\square 0,0220-0,0303$

口 0,0303-0,1065

$\square$ No incluídos

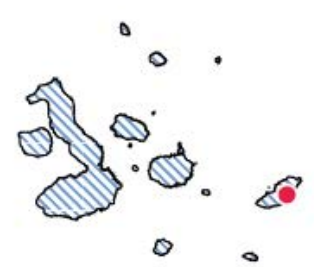

$\square 4,10-6,70$

$\square 6,70-7,40$

$\triangle 7,40-8,10$

$\square 8,10-8,84$

- 8,84-12,40

$\square$ No incluídos

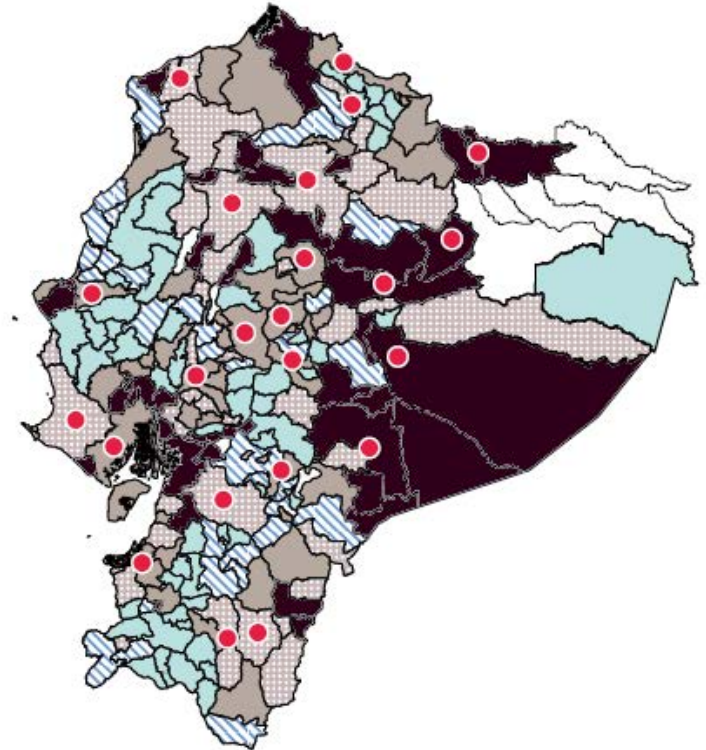

D. Capital, 2007

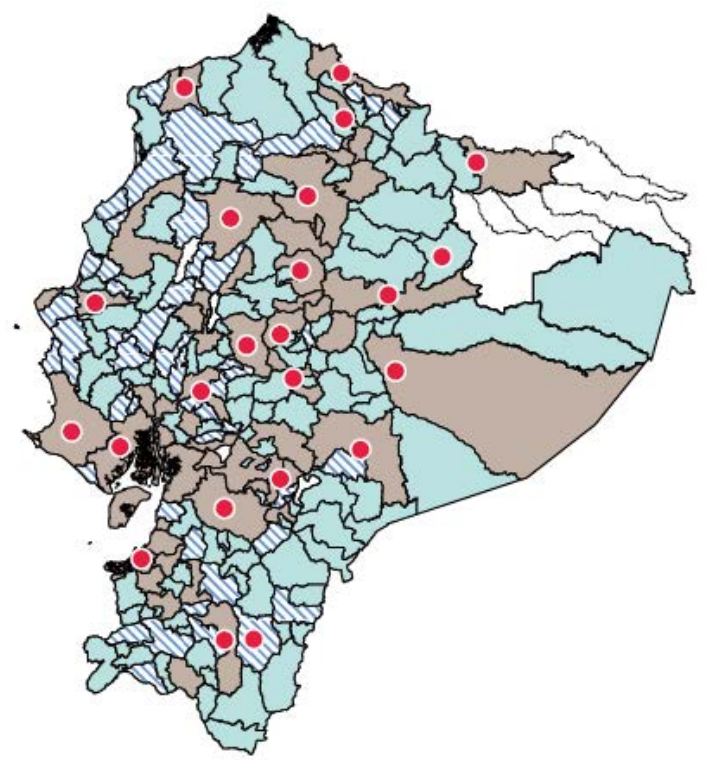


E. Años promedio de asistencia a la escuela, 2010 (educación)

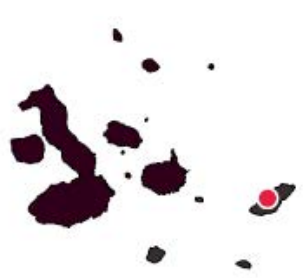

$\square 4,10-6,70$

⿶ $6,70-7,40$

$\square 7,40-8,10$

$\square 8,10-8,84$

口 8,84-12,40

$\square$ No incluídos

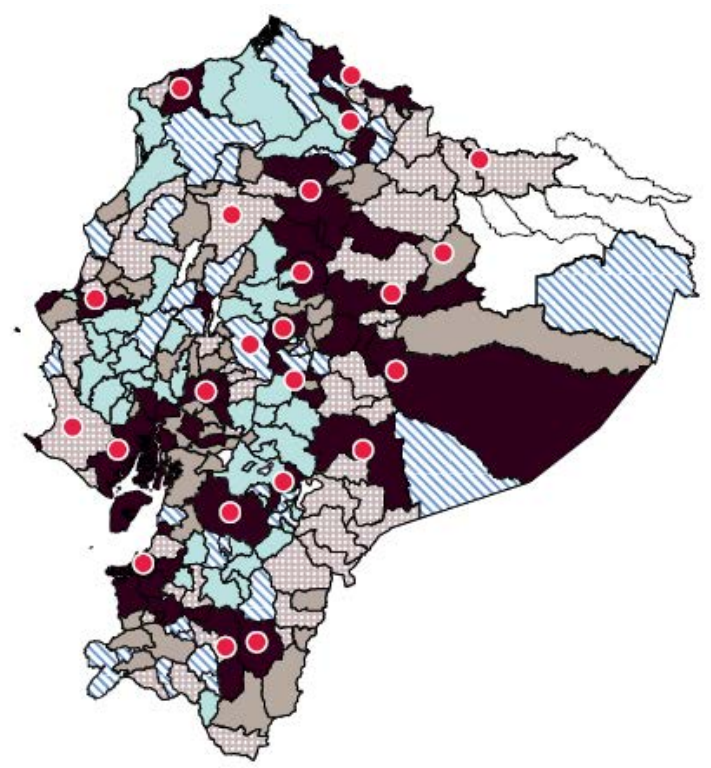

Fuente: Elaboración propia.

Nota: Los puntos representan las capitales provinciales. 


\title{
Anexo A3
}

\author{
Mapa A3.1
}

Índice de Moran local de los coeficientes

A. Índice de Moran local del intercepto
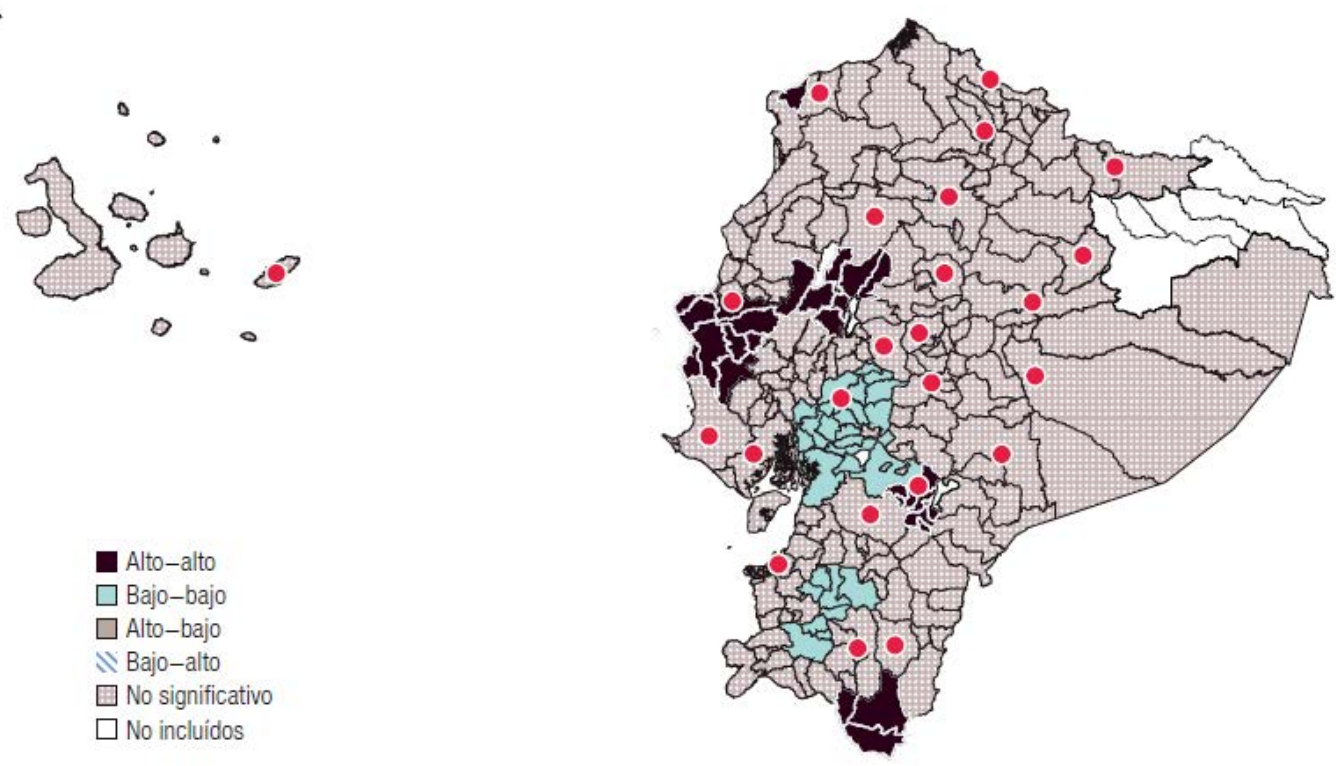

B. Índice de Moran local de la tasa de convergencia

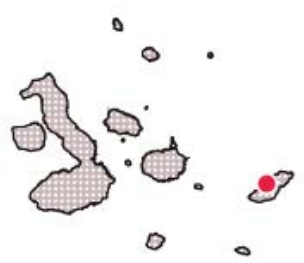

Alto-alto

$\square$ Bajo-bajo

$\square$ Alto-bajo

N Bajo-alto

$\square$ No significativo

$\square$ No incluídos

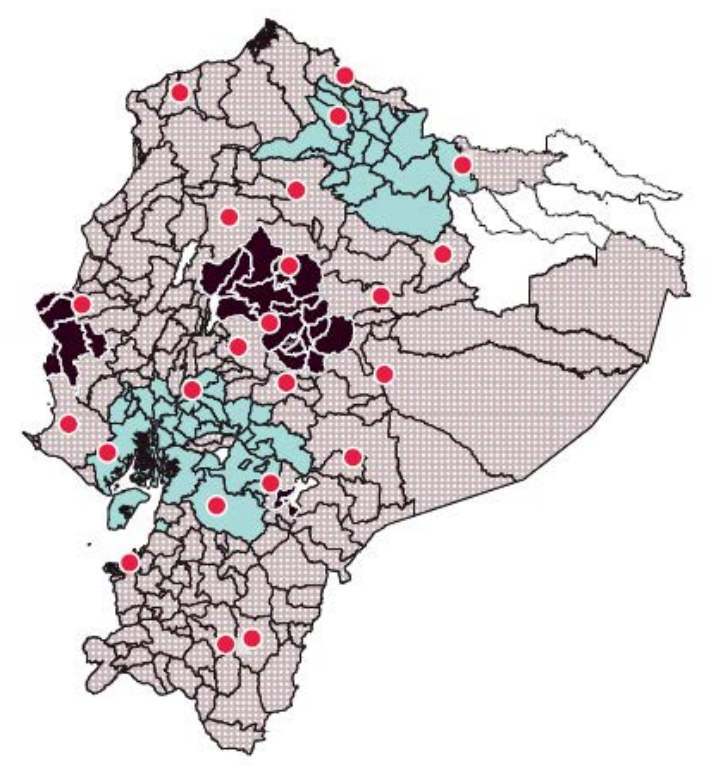


C. Índice de Moran local del coeficiente de la población
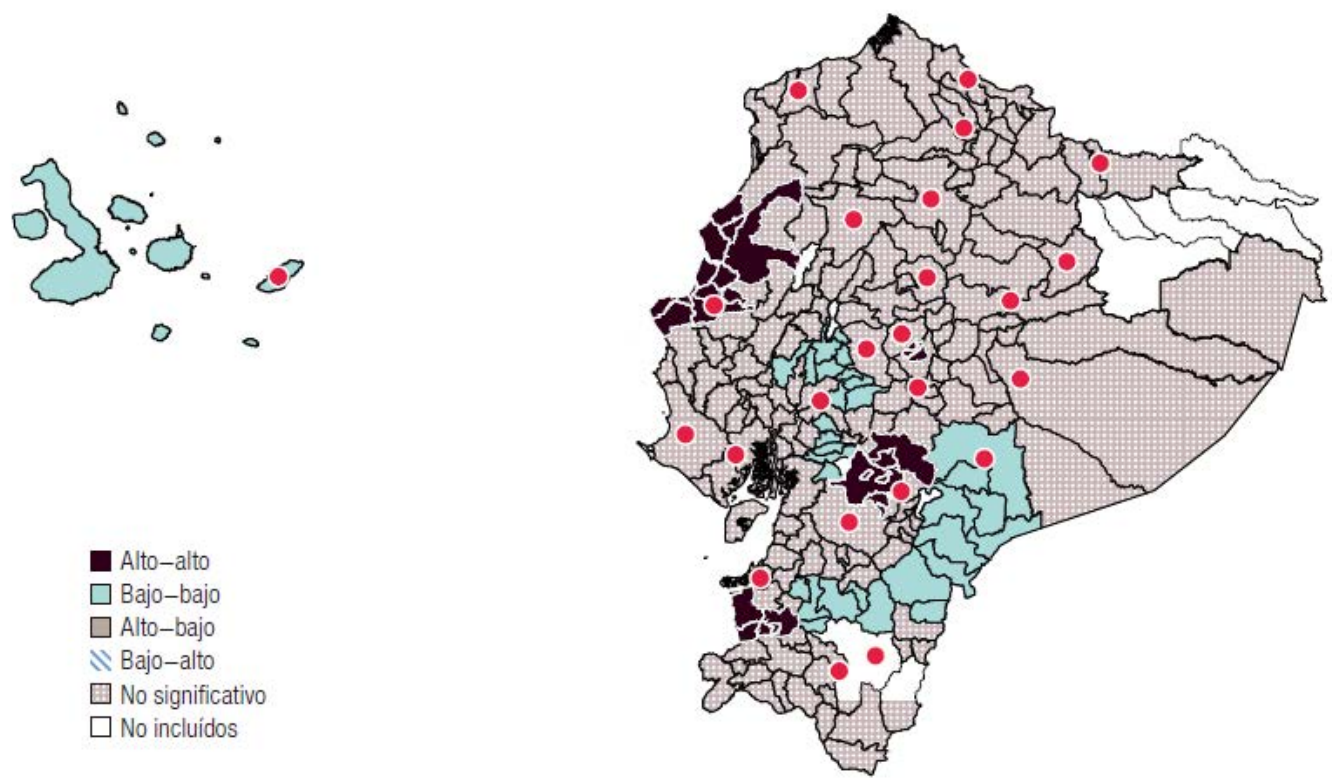

D. Índice de Moran local del coeficiente de capital
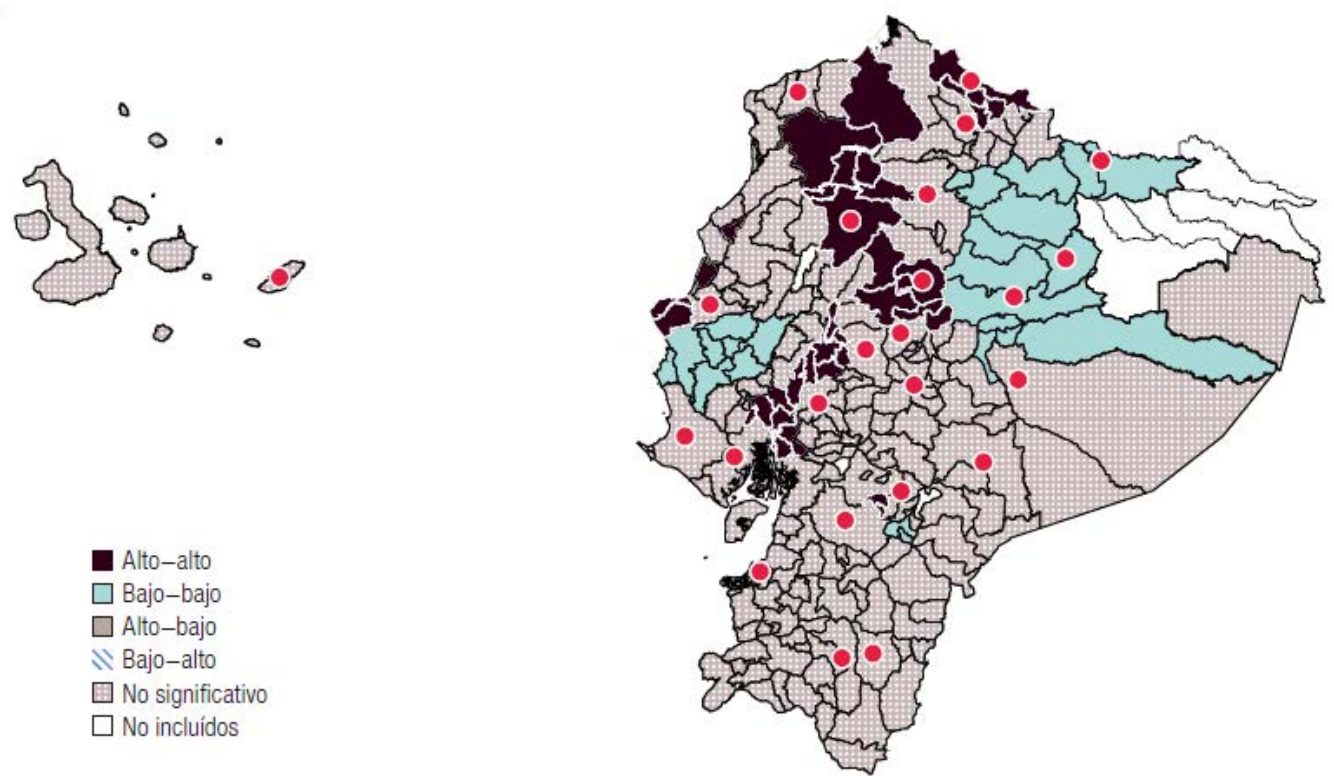
E. Índice de Moran local del coeficiente de la educación
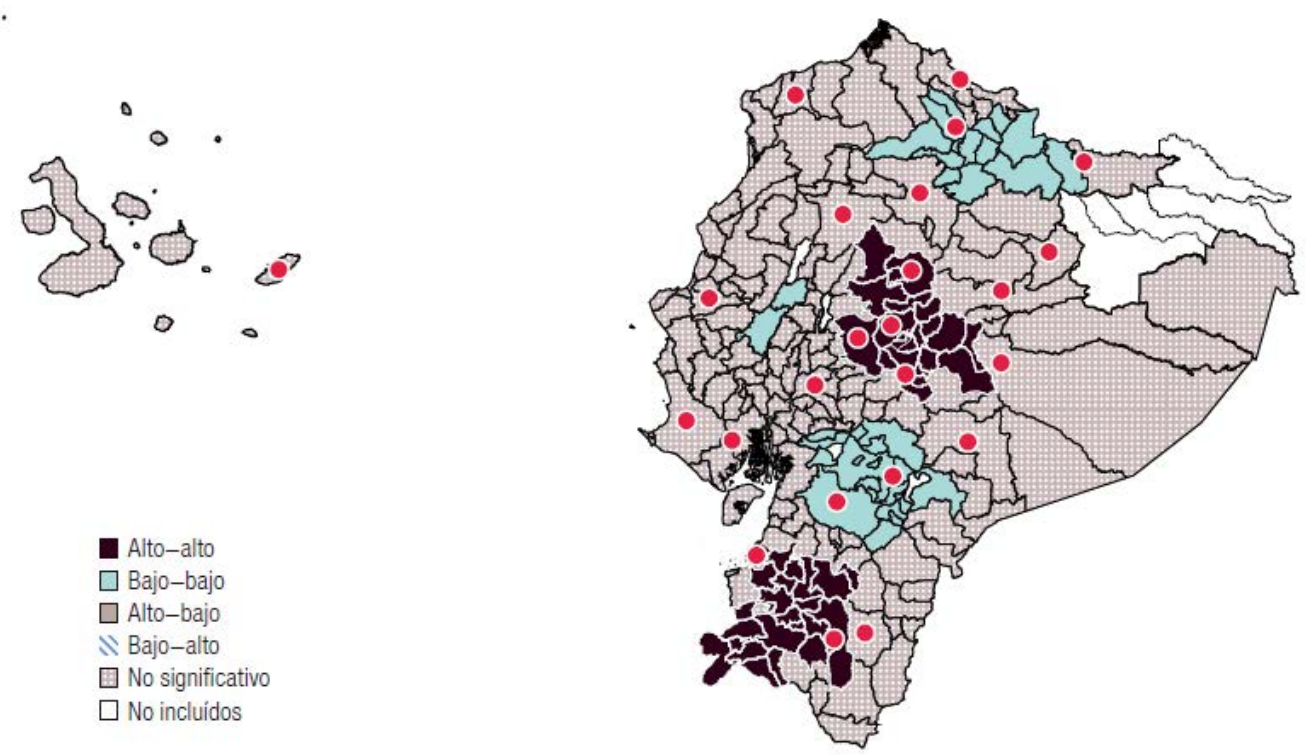

Fuente: Elaboración propia.

Nota: Los puntos representan las capitales provinciales. 